\title{
3-D Nonlinear Evolution of MHD Instabilities
}

\author{
Glenn Bateman \\ H. R. Hicks \\ J. W. Wooten
}

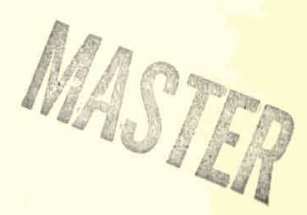

\section{OAK RIDGE NATIONAL LABORATORY}




\section{DISCLAIMER}

This report was prepared as an account of work sponsored by an agency of the United States Government. Neither the United States Government nor any agency Thereof, nor any of their employees, makes any warranty, express or implied, or assumes any legal liability or responsibility for the accuracy, completeness, or usefulness of any information, apparatus, product, or process disclosed, or represents that its use would not infringe privately owned rights. Reference herein to any specific commercial product, process, or service by trade name, trademark, manufacturer, or otherwise does not necessarily constitute or imply its endorsement, recommendation, or favoring by the United States Government or any agency thereof. The views and opinions of authors expressed herein do not necessarily state or reflect those of the United States Government or any agency thereof. 


\section{DISCLAIMER}

Portions of this document may be illegible in electronic image products. Images are produced from the best available original document. 


\section{Printed in the United States of America Availahle from National Technical Information Service U.S. Department of Commerce 5285 Port Royal Road, Springfield, Virginia 22161 Price: Printed Copy $\$ 4.50$; Microfiche $\$ 3.00$}

This report was prepared as an account of work sponsored by the United States Government. Neither the United States nor the Energy Research and Development Administration/United States Nuclear Regulatory Commission, nor any of their employees, nor any of their contractors, subcontractors, or their employees, makes any warranty, express or implied, or assumes any legal liability or responsibility for the accuracy, completeness or usefulness of any information, apparatus, product or process disclosed, or represents that its use would not infringe privately owned rights. 
Contract No. W-7405-eng-26

Fusion Energy Division

3-D NONLINEAR EVOLUTION OF MHD INSTABILITIES

G.lenn Bateman, H. R. Hicks, and J. W. Wooten

Date Published - March 1977

\author{
Prepared by the \\ OAK RIDGE NATIONAL LABORATORY \\ Uak Kidge, Tennessee 37830 \\ operated by \\ UNION CARBIDE CORPORATION \\ for the \\ ENERGY RESEARCH AND DEVELOPMENT ADMINISTRATION
}

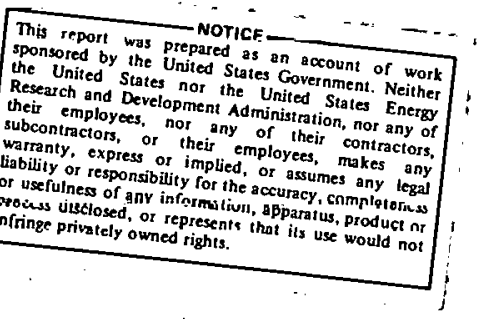




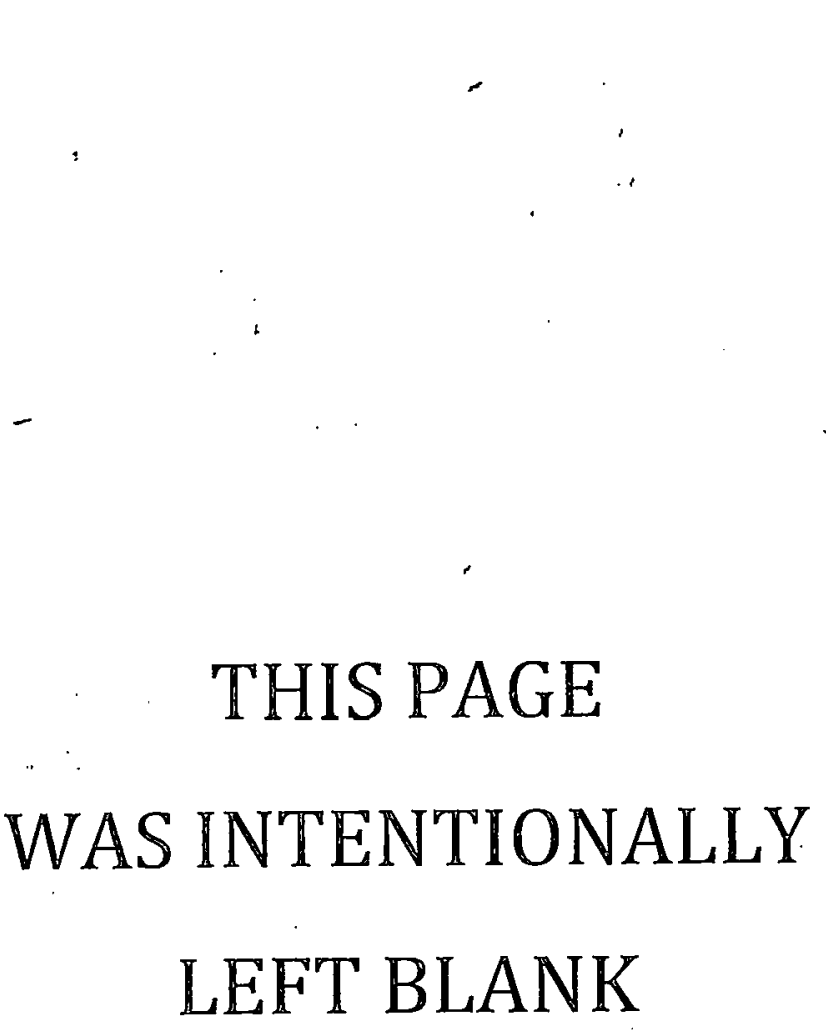




\section{CONTENTS}

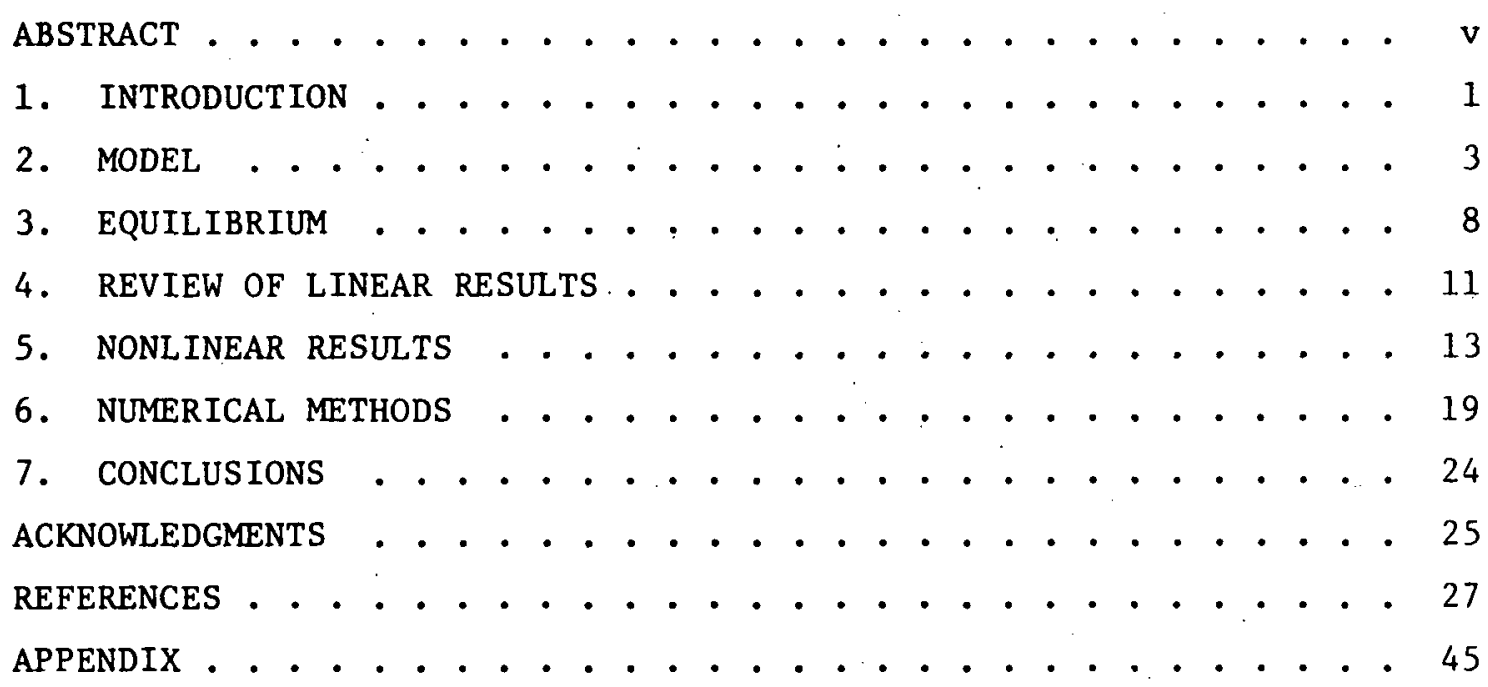




\section{THIS PAGE}

WAS INTENTIONALLY

LEFT BLANK 
The nonlinear evolution of ideal MHD internal instabilities is investigated in straight cylindrical geometry by means of a 3-D initialvalue computer code. These instabilities are characterized by pairs of velocity vortex cells rolling off each other and helically twisted down the plasma column. The cells persist until the poloidal velocity saturates at a few tenths of the Alfúen velocity. The nonlinear phase is characterized by convection around these essentially fixed vortex cells. For example, the initially centrally peaked temperature profile is convected out and around to form an annulus of high temperature surrounding a small region of lower temperature. Weak, centrally localized instabilities do not alter the edge of the plasma. Strong, large-scale instabilities, resulting from a stronger longitudinal equilibrium current, drive the plasma against the wall. After three examples of instability are analyzed in detail, the numerical methods and their verification are discussed. 
Over the past few years we have developed a series of computer. codes [1-12] designed to investigate large-scale internal instabilities by solving the ideal MHD equations as an initial boundary-value problem. Starting with an unstable equilibrium and an arbitrary initial perturbation, the primitive variables (velocity, magnetic field, pressure, and density) are advanced in time until the fastest growing instability dominates over all other motion. The nonlinear evolution of the instability is then followed on a three-dimensional grid until the plasma has convected considerably and the poloidal velocity field saturates. In this paper, the results of three examples and the verification of the numerical methods will be analyzed in detall.

This work is motivated by the fact that large-scale instabilities are observed in all tokamaks $[13,14]$. Some level of unstable activity is observed even during the normal operation of most tokamaks, as is evidenced by the fact that fluctuations are routinely observed in the poloidal magnetic field $[15,16,17]$ and in soft $x$-rays $[18,19]$. Tokamaks operating at such a low level of current density that they appear to be completely free of instabllities [14] are less interesting candidates for thermonuclear fusion than tokamaks with some level of unstable activity. The experimental evidence indicates that some helical structures, associated with large-scale instabilities, saturate at such a low amplitude that they enhance transport, alter the profile, or have hardly any effect at all. However, when the current and mass density are varied beyond a limited range, a disruptive instability appears suddenly and unpredictably $[13,20]$, from which the plasma may or may not recover. Given the dominant role which instabilities play in tokamaks, it is more useful to study the effects of instabilities when they exist than to continue the search for completely stable equilibria.

Sawtooth oscillations observed with the soft $x$-ray diagnostic occur under conditions for which an ideal MHD $m=1$ instability may be expected. The resistive form of the $m=1$ instability has been studied by Waddell et al. [21] in the limit of zero beta. The present paper is devoted to the complementary problem of the ideal MHD $m=1$ instability 
with finite beta. We have investigated these instabilities for a variety of equilibrium profiles and shapes [1-12]. In this paper, three examples will be studied in detall in order to represent our typical results. The equilibrium for these three examples is characterized by a sinusoidally peaked current profile, high beta, and straight cylindrical geometry.

The basic features of the model and computational method are described in Section 2. The equilibrium used for the examples in this paper is described in Section 3 followed by a review of the linear instability results in Section 4. Three examples of nonlinear instability evolution are analyzed in Section 5 . The numerical methods and verification tests are given in Section 6. 
2. MODEL

We have simulated the nonlinear evolution of MHD instabilities by solving the ideal MHD equations as an initial boundary-value problem. The equations are advanced in time by using an explicit leapfrog difference scheme on a 3-D rectangular Cartesian grid. Starting with an unstable equilibrium and adding a small perturbation (usualiy the eigenfunction corresponding to the fastest growing linear instability obtained from a linear code) we follow the evolution of the primitive variables (velocity $v$, magnetic field $B$, pressure $p$, and density $\rho$ ) for as long as the numerical procedure is réliable. For a given equilibrium, the results follow a consistent pattern regardless of the initial perturbation, provided the perturbation is sufficientiy small.

The ideal MHD equations are written in an almost conservative form in Cartesian coordinates: , : ...

$$
\begin{aligned}
& \frac{\partial}{\partial t}\left(\rho v_{i}\right)=\frac{\partial}{\partial x_{j}}\left[-\rho v_{i} v_{j}+B_{i} B_{j}-\left(p+\frac{1}{2} B^{2}\right) \cdot \delta_{i j}\right] \\
& \frac{\partial}{\partial t} \underset{\sim}{B}=-\nabla \times \underset{\sim}{E}, \underset{\sim}{E}=-\underset{\sim}{\mathbf{V}} \times \underset{\sim}{B} \\
& \frac{\partial}{\partial t} \rho=-\nabla \cdot \underset{\sim}{(\rho)} \underset{\sim}{v} \\
& \begin{array}{rrr}
\because \cdots & \therefore \\
& \therefore \quad \therefore
\end{array} \\
& \frac{\partial}{\partial t} p=-\nabla \cdot(p v)-(\Gamma-1) p \nabla \cdot v, \quad \Gamma \cdot=5 / 3
\end{aligned}
$$

These equations apply throughout the domain - there is no vacuum region. The boundary conditions are chosen to isolate the system by globally. conserving energy, magnetic fluxes,: and total mass. The conditions used at the walls are:

$$
\underset{\sim}{\mathrm{E}_{\|}}=0
$$




$$
\begin{aligned}
& \mathrm{v}_{1}=0 \\
& \mathrm{p}=0 \\
& \underline{B}_{1}=0
\end{aligned}
$$

These conditions are consistent with the finite difference form of the equations used. The variables $\underline{\sim}_{\|}, \underline{B}_{\|}$, and $\rho$ are time advanced on the boundary in a way which will be discussed in Section 6 .

Equation (2.4) could be rewritten in conservative form by replacing it with an equation for the evolution of the energy density:

$$
\frac{\partial}{\partial t}\left(\frac{1}{2} \rho v^{2}+\frac{p}{\gamma-1}+\frac{1}{2} B^{2}\right)=-\nabla \cdot\left[\left(\frac{1}{2} \rho v^{2}+\frac{\gamma p}{\gamma-1}\right) \underset{\sim}{v}+\underset{\sim}{E} \times \underset{\sim}{B}\right]
$$

The kinetic and magnetic energy may be eliminated whenever $p$ is needed. Equation (2.4) was used instead because it is simpler and it avoids round-off error (the small difference between large numbers) when the kinetic or magnetic energy is much larger than the pressure. Several rearrangements of Eq. (2.4) were tried and had no noticeable effect on the results.

The equations are written in dimensionless form by normalizing

1) all lengths by the minor radius $a$,

2) density by the initial density at the magnetic axis $\rho_{0}$,

3) magnetic fields by the initial longitudinal vacuum magnetic field at the center of the domain, $B_{\text {To }}$

These three normalization parameters $\left(a, \rho_{0}, B_{T_{0}}\right)$ are then used to define reference values for the Alffen velocity, $v_{A O} \equiv B_{\text {TO }} /\left(4 \pi \rho_{0}\right)^{\frac{1}{2}}$; pressure, $\dot{\mathrm{P}}_{\mathrm{O}} \equiv \mathrm{B}_{\text {To }}{ }^{2} / 4 \pi=\mathrm{v}_{\mathrm{AO}}{ }^{2} \rho_{\mathrm{O}}$ (not related to the central plasma pressure); time, $t_{0} \equiv \mathrm{a} / \mathrm{v}_{\mathrm{Ao}}$; current density, $\mathrm{J}_{0} \equiv \mathrm{cB}_{\mathrm{To}} / 4 \pi \mathrm{a}$; and electric field $E_{o} \equiv v_{A o}{ }^{B} T_{0} / c$, using Gaussian units here. For example, the conversion of growth rate and current density to practical units is given by 


$$
\begin{gathered}
\gamma\left[\mu \mathrm{sec}^{-1}\right]=\frac{2.18 \times 10^{8} \mathrm{~B}[\mathrm{kG}] Y}{\mathrm{a}[\mathrm{cm}]\left(\mathrm{n}\left[\mathrm{cm}^{-3}\right] \mathrm{m}_{1} / \mathrm{m}_{\mathrm{p}}\right) \frac{1}{2}} \\
\mathrm{~J}\left[\mathrm{Amp} / \mathrm{cm}^{2}\right]=795.8 \frac{\mathrm{B}[\mathrm{kG}]}{\mathrm{a}[\mathrm{cm}]} \mathrm{J}
\end{gathered}
$$

where $m_{1}$ is the Ion mass, $m_{p}$ is the proton mass, and $n$ is the ion number density.

When using the MHD codes, our standard procedure is to first prepare an equilibrium, then find the fastest growing instability using the linearized MHD equations, and finally follow the evolution of the instability. using the nonlinear MHD equations. We could dispense with the step of finding an instability from the linearized equations and simply use an arbitrary initial perturbation added to the equilibrium. We could also dispense with the preparation of an equilibrium and start the nonlinear evolution with an arbitrary plasma state.

We start with the sum of an equilibrium and an eigenfunction of the linearized equations for several reasons:

1) The eigenfunctions of the linearized equations are unique and independent of the initial perturbation. Hence, for any given equilibrium, we are assured of a unique nonlinear evolution if we start with a perturbation which is initially so small that it evolves according to the linearized equations long enough for the fastest growing eigenfunction to dominate over all other motion. If this procedure is to be followed, it is economically advantageous to use the linearized equations to determine the fastest growing eigenfunction.

2) We routinely check the nonlinear code by comparing the evolution at small levels of the perturbation against the growth rates and eigenfunctions obtained from the linearized code. In order to minimize any initial transient, the difference. schemes used in the codes have been made identical. It has been found that even when the equilibrium is not perfect or the codes are not identical, the growth rate and form of the eigenfunction agree to within several percent of the linear results. 
3) Our intuition is based upon the linear results. We feel it would be more useful to extend this body of knowledge into the realm of nonlinear evolution than to develop a new and separate set of observations based on the nonlinear evolution of arbitrary finite perturbàtions.

A possible disadvantage of always starting with the fastest growing eigenfunction of the linearized equations is that we might miss those nonlinear effects which depend upon the coupling of two or more eigenfunctions or upon perturbations which cannot be represented as the sum of those eigenfunctions which are available to us (on the grid we use). In answer to these objections, we have tried some computer runs with bizarre initial perturbations which excited a variety of MHD oscillations. During the nonlinear evolution, we observed that the fastest growing instability always emerges from the background motion before the typical nonlinear effects take place. This happens even with unusually large Initial perturbations.

We have chosen to advance the equations in time by using an explicit leapfrog finite difference scheme $[22,23]$ on a 3-D Cartesian grid because it is the simplest numerical method with second-order accuracy. The numerical instability characterized by grid separation does not appear as long as the variables are growing in time. This will be discussed further in Section 5 .

We must avoid equilibria with values of beta lower than a few percent for three reasons:

1) Low beta instabilities tend to be spatially discontinuous or localized. For example, the radial velocity for the $m=1$ internal kink mode in a circular cylinder looks like a step function at the radius of the mode-rational surface and the $m \geqslant 2$ modes are localized near the rädii of their respective mode-rational surfaces in the limlt of very low beta and conditions near marginal stability [24]. With high beta and finite growth rates, the instabilities are smoother and broader and therefore better approximated by a discrete grid.

2) Low beta internal instabilities grow very slowly on the Alfúen transit time scale. Hence the lower the beta, the longer our code would need to run in order to follow an instability for several e-folding times. 
3) Finally, low beta instabilities are driven by a small difference between large forces. Not only would they take longer to grow, but they would need more numerical accuracy to approximate. 


\section{EQUILIBRIUM}

A wide variety of equilibrium shapes and profiles has been investigated with both the linear and nonlinear MHD instability codes. However, a standard analytic equilibrium will be used for the three examples considered in this paper.

The static equilibrium filling a straight cylinder with square cross section (Fig. 1) is specified by

$$
\begin{aligned}
& \mathrm{p}^{\prime}(\psi)=J_{\mathrm{c}} \psi / \psi_{\mathrm{c}}=-\nabla^{2} \psi \\
& \mathrm{B}_{\mathrm{T}}(\mathrm{x}, \mathrm{y})=1 \\
& \rho(\mathrm{x}, \mathrm{y})=1
\end{aligned}
$$

where $\psi$ is the stream function, proportional to poloidal flux, from which the poloidal magnetic field is determined such that

$$
\underset{\sim}{\mathrm{B}}=\nabla \times(\psi \nabla \mathrm{z})+\mathrm{B}_{\mathrm{T}} \hat{\mathrm{Z}}
$$

and where $\psi_{c}$ and $J_{c}$ are the central value of $\psi$ and the longitudinal current density (along $Z$ ). The wall is a flux surface on which $\psi=0$ and $p=0$. There are no poloidal currents $\left(\beta_{\text {poloidal }}=1\right)$.

The analytic solution for this equilibrium is given by

$$
\psi=\psi_{c} \cos (\pi x / 2 a) \cos (\pi y / 2 b)
$$

where $a$ and $b$ are the half-width and half-height of the rectangular walls $(a=b=1$ here $)$ and

$$
\psi_{c}=J_{c} /\left[\left(\frac{\pi}{2 a}\right)^{2}+\left(\frac{\pi}{2 b}\right)^{2}\right]
$$




$$
p(x, y)=\frac{1}{2} J_{c} \psi^{2}(x, y) / \psi_{c}
$$

The current profile, $J_{T}(x, y)=j_{C} \psi / \psi_{c}$, is essentially parabolic.

A longitudinal periodicity length is specified for the cylinder in the 3-D nonlinear code matching the wavelength of the linear instability used as the initial perturbation. The product form of the terms in the nonlinear equations assures that only subharmonics of this wavelength will be generated $(n k, n=0,1,2, \ldots$ where $k$ is the wavenumber of the initial perturbation) so that periodic end conditions remain consistent. The q-value per unit wavelength at the magnetic axis is given by $[24]$

$$
\mathrm{nq}_{c}=\frac{\mathrm{B}_{\mathrm{T}}}{\star\left(\left|\frac{\partial \mathrm{By}}{\partial \mathrm{x}}\right|\left|\frac{\partial \mathrm{Bx}}{\partial \mathrm{y}}\right|\right)^{\frac{1}{2}}}
$$

where $\dot{\lambda}$ is the wavelength divided by $2 \pi(\lambda=1 / k)$. When the flux surfaces at the magnetic axis are circular, this reduces to

$$
\mathrm{nq}_{\mathrm{c}}=\frac{2 \cdot \mathrm{B}_{\mathrm{Tc}}}{\lambda \mathrm{J}_{\mathrm{c}}}
$$

The q-value at the edge is infinite because the poloidal magnetic field has stagnation points in the corners of the rectangular walls. Plots of q-value as a function of the radius along the midplane of the cylinder cross section are shown in Fig. 2 for the three cases to be studied in this paper. The q-value at an arbitrary point is given by

$$
q(\psi)=q_{c} \frac{2}{\pi} K\left[1-\left(\psi / \psi_{c}\right)^{2}\right]
$$

where $\mathrm{K}$ is a complete elliptic integral of the first kind [26].

The maximum value of beta, using the definition

$$
B_{(x, y)} \equiv \frac{p}{p+\frac{1}{2} B^{2}}
$$


is the value at the center given by

$$
\beta_{\max }=\left[1+\left(a^{2}+b^{2}\right) \pi^{2} B_{T c}{ }^{2} / 4 a^{2} b^{2} J_{e^{2}}\right]^{-1}
$$

For comparison with experiments, it is useful to quote the average toroidal beta

$$
\beta_{\text {ave }} \equiv: \frac{\langle\mathrm{P}\rangle}{\frac{1}{2} \mathrm{~B}_{\mathrm{T} c}^{2}}=1 / 2 \frac{\mathrm{P}_{\max }}{\mathrm{B}_{\mathrm{T} c}^{2}}
$$

which is roughly a quarter of $\beta_{\max }$ for this equilibrium in the low- $B$ limit.

A short cylinder, $\lambda=1$, and low central q-values, $\mathrm{nq}_{\mathrm{c}}=0.6,0.95$, and 1.6, are used for the results presented in this paper. See Table 1 for a full list of conditions. The nonlinear instability code produces the most accurate and reliable results under these conditions of high $B$ short wavelength and broad current profile. Only qualitative agreement can be expected with tokamak or shock heated experiments. Results for longer wavelengths, lower $\beta$, more peaked profiles, $\beta_{\text {poloidal }} \neq 1$, and different geometries will be presented in future reports. 


\section{REVIEW OF IINEAR RESULTS}

What follows is a brief review of the results for linear fixedboundary instabilities in a straight cylinder [1].

These instabilities are characterized by pairs of velocity vortex cells rolling off each other and hellcally twisted down the cylinder. The number of vortex cells arranged azimuthally around the magnetic axis (the azimuthal mode number $\mathrm{m}$ ) depends upon the equilibrium parameters and the wavelength along the cylinder. In the case of a circular cyiinder, it is a theorem [27] that modes with two or more vortices arranged radially from the magnetic axis always grow more slowly than the mode with only one radial vortex cell for any given azimuthal mode number. We have observed only the fastest growing modes in the square cylinder and each of the modes observed has only one radial vortex cell.

The spatial extent of the velocity pattern depends upon the breadth of the profile and the strength of the instability. For centrally peaked current profiles, the instability concentrates near the magnetic axis as the current is reduced to the low-current marginal point and it concentrates near the wall as the current is increased to the highcurrent marginal point. Roughly speaking, the instability is strongest Inside and near the mode rational surface. Between the marginal points and near the maximum growth rate, the instability has a broad spatial extent completely filling the cylinder and there is a strong longitudinal velocity which is maximum at the outer edges of the vortex cells. This velocity is driven by the gradient of the perturbed pressure parallel to the magnetic field lines wherever the helicity of a line of constant pressure does not match the helicity of the local field lines.

The perturbed pressure responds to the velocity field by forming $\mathrm{m}$-positive maxima and $\mathrm{m}$-negative minima arranged azimuthally around the magnetic axis and helically twisted down the plasma column.

The perturbed magnetic field and perturbed current density consist of 2-m helically twisted vortex cells, with regions of longitudinal component near the center of each vortex cell, $+90 / \mathrm{m}^{\circ}$ and $-90 / \mathrm{m}^{\circ} \mathrm{respec}-$ tively, out of phase with the velocity vortex cells. 
Growth rate curves as a function of central q-value are shown in Fig. 3 for the $m=0,1,2$, and 3 modes with wavelengths 1,2 , and 3 in units of $2 \pi a$. Corresponding growth rate curves for a circular cylinder with parabolic current density are also shown for comparison. These circular cylinder curves were obtained from a shooting code similar to those used in Refs. [27 and 28]. The remarkably close agreement of the curves indicates that the details of the geometry have little effect on these large-scale instabilities. The fact that the curves differ at the low-current (high q-value) marginal points is probably indicative of the inaccuracy of the initial-value code (the one used for the square cylinder) as the mode concentrates near the magnetic axis. This is especially true for the higher mode numbers. In Fig. 3, arrows point to the three cases whose nonlinear behavior is studied in this paper. The linear growth rates for these three cases agree well with the circular cylinder results. 


\section{NONLINEAR RESULTS}

We find that the nonlinear evolution of large-scale internal Instabilities is characterized by convection around essentially fixed velocity vortex cells. The poloidal velocity field established by the linear instability continues to grow long into the nonlinear phase without appreclably changing structure or wandering. The form of the linear instability is indicative of the nonlinear behavior, in this sense. The effects of convection can then be seen in the evolution of temperature, pressure, density, magnetic field, and current density. Compression and other driving terms also contribute to the evolution of these quantities.. In each case, the physical quantities grow exponen-. tially at first, consistent with the linear MHD results. As the maximum poloidal velocities grow to a few tenths of the Alfien velocity and appreciable convection occurs, the growth rates decrease to zero and the instability appears to saturate.

It is useful to draw a distinction between instabilities which are concentrated near the center of the plasma and those which extend over most of the cross section. The extent of the poloidal velocity field is a good indicator of this localization. Under conditions where the instability is centrally localized, i.e., conditions near the lowcurrent marginal point, the resulting noniinear convection is also centrally localized leaving the edge of the plasma essentially untouched. Extreme cases of localization are difficult to simulate accurately because a fine grid is needed to support the detail. A mildly localized $m=1$ instability, for which the central q-value is 0.95 , is illustrated by the time sequences in Fig. 4 corresponding to Case I in Table 1.

An instability with broad spatial extent drives the plasma into the wall. Nothing actually passes through the wall in this model but the hot core of the plasma is squeezed up so close to the wall that transport effects, which are not included in the MHD model, are likely to be large. An example of a broad $m=1$ instability leading up to this state, for which the central equilibrium q-value is 0.6 , is illustrated by the time sequences in Fig. 5, corresponding to Case II in Table 1. 
Table 1

\begin{tabular}{|c|c|c|c|}
\hline & Case $I^{a}$ & Case II & Case TIT \\
\hline nq central & 0.95 & 0.60 & 1.60 \\
\hline $\mathrm{J}_{\mathrm{c}}$ & 2.105 & 3.333 & 1.25 \\
\hline$\beta_{\max }$ & 0.47 & 0.69 & 0.24 \\
\hline$\beta_{\text {ave }}=\langle\mathrm{P}\rangle / \frac{1}{2} \mathrm{~B}_{\mathrm{TC}}^{2}$ & 0.22 & 0.56 & 0.079 \\
\hline m & 1 & 1 & 2 \\
\hline$\gamma_{\text {linear }}$ & 0.240 & 0.535 & 0.117 \\
\hline Pololdal saturation velocity & 0.14 & $>0.5$ & 0.095 \\
\hline $\begin{array}{l}\text { Radius of mode-rational } \\
\text { surface }\end{array}$ & 0.29 & 0.8 & 0.57 \\
\hline Radius of vortex center & 0.29 & 0.51 & $0.29(x \sqrt{2})$ \\
\hline Radius where & . & & \\
\hline$v_{y}=\frac{1}{2} v_{y \max }$ & 0.35 & .0 .58 & 0.52 \\
\hline Radius of $\mathrm{P}_{\max }^{1}$ & 0.29 & 0.33 & 0.36 \\
\hline Radius of $B_{\text {TOR max }}^{l}$ & 0.29 & 0.46 & 0.38 \\
\hline
\end{tabular}


The nonlinear consequences of an $m=2$ instability are illustrated in Fig. 6, corresponding to Case III in Table 1. A fine grid is needed to support the detalled structure of an $m=2$ instability. The grounds for confidence in the particular example chosen $\left(\mathrm{nq}_{\mathrm{c}}=1.6\right)$ are optimal in the sense that the square cylinder growth rate agrees very well with the circular cylinder result (see Fig. 3) and the structure is moderately broad, In addition to the fact that much the same behavior is observed when a different grid is used (e.g., $24 \times 24 \times 20$ rather than $27 \times$ $\left.27^{\circ} \times 16\right)$.

Relevant parameters for all three cases are presented in Table 1. Note that the values of beta and current density are high because a short wavelength is being used $(\lambda=2 \pi a)$. The last four radii given in the table characterize the spatial extent of the instability. Perturbation quantities are indicated by the superscript 1 while equilibrium quantities are indicated by the superscript 0 .

Figures 4,5 , and 6 show time sequences for a cross section of velocity, pressure, perturbed magnetic field $\left(\dot{B}-B^{\circ}\right)$, perturbed poloidal current density $\left[\left(\mathrm{J}-\mathrm{J}^{\circ}\right){ }_{\mathrm{pol}}\right]$, rate of change of velocity $(\partial \mathrm{v} / \partial \mathrm{t})$, divergence of velocity $(\nabla \because v)$, longitudinal current density $\left(\mathrm{J}_{z}\right)$, and mass density $(\rho)$. Each frame represents a snapshot for the time given at the top of each illustration (in units of the reference Alfven time). The structure shown in these plots is helically twisted down the cylinder in the sense of a right-handed screw along the positive z-axis (out of the page). For the $m=1$ modes, the plots should be turned $90^{\circ}$ counterclockwlse for a representation of each successive quarter-wavelength cross section. For the $\mathrm{m}=2$ mode, they should be turned $90^{\circ}$ counterclockwise for each half-wavelength cross section. To a good approximation, any structure near the center can be rotated continuously to obtain intermediate cross sections.

See Appendix I for the information needed to interpret these contour plots and arrow plots.

In all three cases it is clear that the evolution of the pressure contours is dominated by convection around the velocity vortex patterns. The effects of convection are also evident as the structure of the 
perturbed magnetic field and current density evolve in time while their magnitudes grow exponentially. The poloidal velocity continues to grow in magnitude but the structure and the position of the vortex cells do not change appreciably until a considerable amount of convection has occurred.

Since the spatial form of the poloidal velocity field does not change substantially during most of the evolution, it is useful to measure the magnitude of the poloidal velocity at a fixed point in space. Figures 7,8 , and 9 show log-linear plots of the maximum value of $v_{y}$ along the vertical midplane as a function of time for the three cases under consideration. Also shown are plots of the kinetic energy integrated over the total volume and the decrement in the total energy (kinetic plus magnetic plus thermodynamic) as a function of time. Arrows indicate the instants in time which are illustrated in Figs. 4, 5 , and 6 .

In all three cases, the poloidal velocity shows signs of saturating at a few tenths of the reference Alfven velocity. The stronger the instability, the larger the saturation velocity. The kinetic energy continues to rise after the growth of the poloidal velocity has saturated. Evidently the additional kinetic energy comes from longitudinal velocity which, in turn, appears to be due to gradients of pressure which build up along magneric field lines.

The fact that the energy decrement, which is purely a numerical artifact, is typically an order of magnitude smaller than the kinetic energy adds confidence to the results. This decrement is several orders of magnitude smaller than the total energy.

Relurning to Figg. 4, 5, and 6, we note that the maximum pressure does not change drastically during the evolution. However, the effects of local compression and expansion $(\nabla \cdot v \neq 0$, which is shown in the sixth row of each sequence) are enough to change the topology of the isobars, especially when a narrow ridge forms. Th1s can be estimated by noting that the pressure (or any quantity) is constant in a fluid element under the effect of convection alone. It is sufficient to integrate $\nabla \cdot v$ over time to estimate the change in magnitude due to expansion. 
More than a $20 \%$ change, for example, would be expected for the bridge connecting the two pressure maxima in the last frame shown in Case $\mathrm{I}$. (Fig. 4).

In order to understand the evolution of the magnetic field as a whole, first consider the evolution of the total perturbation of the magnetic field from equilibrium $-B^{l} \equiv B-B^{\circ}$. As shown in Figs. 4, 5 , and 6 , the perturbed magnetic field consists of pairs of longitudinal: bundles each surrounded with a roughly vortex-like-structure, oriented in the direction of the left-hand rule, all helically twisted down the cylinder. The perturbed current density has a corresponding vortex and bundle-like structure. The perturbed magnetic field grows in magnitude and convects. As a result, the total longitudinal field increases near the center and decreases near the edge. The net poloidal magnetic field profile is flattened at the mode rational surface, being increased somewhat near the center and decreased near the edge. The new poloidal current is paramagnetic while the additional longitudinal current is diamagnetic near the center.

For the purpose of gaining physical intuition, it is useful to .. write Faraday's law in the following form

$$
\frac{\partial \mathrm{B}}{\partial \mathrm{t}}=-\underset{\mathrm{v}}{\mathrm{v}} \cdot \nabla \underset{\sim}{\mathrm{B}}+\underset{\sim}{\mathrm{B}} \cdot \nabla \underset{\sim}{\mathrm{v}}-\underset{\sim}{\mathrm{B}} \nabla \cdot \underset{\sim}{\mathrm{v}},
$$

(only the conservative form of this equation is used in the computer code). The first term on the right represents convection and the third term gives the effect of compression or expansion.

Consider an $m=1$ mode. The equation for the poloidal component $\left(B_{x}^{l}\right)$ at the center of the domain is

$$
\left.\frac{\partial B^{1}}{\partial t}=v v_{y}^{(1 / 2]^{0}}-k R_{z}\right)-\underset{\sim}{v} \cdot \nabla R_{x}^{1}-B_{x}^{1} \nabla \cdot \underset{\sim}{v}
$$

The condition $\frac{1}{2} \mathrm{~J}_{\mathrm{z}}^{\circ}>\mathrm{kB}_{\mathrm{z}}^{\circ}$ at the center is just the condition $\mathrm{q}<1$. Hence, $B^{l}$ continues to grow with its original form until the nonlinear cerms become important. Of the three nonlinear terms on the right of 
Eq. (5.25), the convection term dominates over the compression term because the perturbed magnetic field has a great deal of spatial structure: For the second term on the right of Eq. (5.25), we note that the total magnetic field does not appear to change in structure; its components change only in magnitude. Since the velocity field also changes little in structure, this term takes the same form as the linear contributions to the evolution of $\mathrm{B}_{x}^{1}$. However, since the magnitude $\mathrm{B}_{z}$. increases at the center, the growth of $\mathrm{B}_{x}{ }_{x}$ decreases and ultimately reverses there.

Now consider the effect of an $m=1$ mode on the longitudinal magnetic field at the center. The middle term in Eq. (5.25) makes no contribution $\left[v_{z}(0,0)=0\right]$, but compression tends to increase $B_{z}$ below the center and convection carries this increased longitudinal field into the center of the domain.

Consider now the time rate of change of the velocity field (fifth row in Figs. 4, 5, and 6). This is a sensitive indicator of changes in the velocity field. It represents the acceleration at fixed points in space. Significant changes take place just before and after the last frame shown in each time sequence. The computer runs continue after this time but the results are less certain in the sense that fine-scale structure rapidly appears (which might be a numerical artifact). The largest component of this structure is always longitudinal.

Moderately large increases in longitudinal current density (seventh row in Figs. 4, 5, and 6) reflect the growth of perturbed poloidal magnetic field components to values comparable to the poloidal equilibrium fields but with finer structure.

Finally, the evolution of the mass density is illustrated (eighth row in Figs. 4, 5, and 6). A uniform equilibrium density was used in order to ohow the cvolution of a ocaler ficld dominated by compreooion. other computer runs have indicated that all the other results are essentially unaffected by the choice of a mildiy peaked density profile. 


\section{NUMERICAL METHODS}

The computer code used in this report time advances the velocity, magnetic field, density, and pressure at each point on a 3-D Cartesian grid using an Eulerian explicit leapfrog difference scheme. The equations are written in conservative form (except for the pressure equation, as explained in Section 2) and the derivatives are centered in time and space to enhance convergence. At any given point, the variables are. known at one instant in time, but that time is different by half a timestep between adjacent grid points. In principle, the evolution is followed until the effects under consideration have run their course. In practice, the timestep is calculated for each step of the iteration and the evolution must be terminated when the timestep begins to change noticeably. A simple leapfrog scheme without added diffusion is suitable while the variables are growing in time, but it breaks down (due to grid separation) when the variables start to decay.

The difference scheme in the linear MHD code, which is used to prepare the eigenfunction perturbation to initiallze the nonlinear code, is almost identical to the difference scheme in the nonlinear code. The variables in the linear code represent one Fourier harmonic along the ignorable coordinate, $\mathrm{Z}$, of the equiltbrium. The real and imaginary parts of this harmonic represent the variables on planes a quarter of a wavelength apart. The two other quarter-wavelength planes follow by periodicity. A derivative of a linearized variable in the $Z$ direction is equivalent to a finite difference. over four planes with $2 \mathrm{DZ}=1 / \mathrm{k}$, . where $k$ is the wavenumber of the Fourier harmonic. . Because the linear and nonlinear codes are so much alike, there is a continuity of growth rate and symmetry for as long as the eigenfunction is a small perturbation of the equilibrium. A transient fluctuation in the growth rate occurs, as illustrated in Fig. 10, for two reasons: there is a finite difference error in the $z$-derivative depending upon the number of planes used in the nonlinear code; and the equilibrium used is not an exact. solution of force balance in finite difference form. This makes the : equilibrium expand or compress toward the center, changing the pressure 
and current profiles and thereby changing the instantaneous growth rate. It is clear from Fig. 10 that the transient becomes negligible as the grid is made finer. Only the finest grid $(27 \times 27 \times 16)$ was used in preparing the results shown in Section 5.

The boundary conditions in a finite difference scheme require special attention. We impose the conditions given by Eqs. $(2.5)-(2.8)$. These conditions are self-consistent in both analytic and finite difference form. However, they represent conditions for only five out of 11 scalar variables (including the components of $\mathrm{E}$ ); the mass density, perpendicular E-field, and parallel components of $\underset{\sim}{v}$ and $\underset{\sim}{B}$ are still free. Analytically, these follow by continuity as they evolve within the domain. But in finite difference form a choice of extrapolation techniques must be made. We have made a special choice, called "marching boundary conditions," in which these nonzero variables are advanced in time and only those variables which are zero on the boundary are extrapolated in order to find their derivatives perpendicular to the boundary. These boundary equations are derived in the following way.

Consider one form of the equation for the parallel component of the velocity at the wall, $\underline{\sim}_{\|}$:

$$
\rho \frac{\partial v_{\sim}}{\partial t}=-\underline{\sim}_{\|} \cdot \nabla{\underset{\sim}{v}}_{\|}-\nabla_{\|} p+(\underset{\sim}{J} \times B)_{\|}
$$

Since $p=0$, its parallel gradient is zero on the wall. Since ${\underset{\sim}{1}}_{1}=0$, only parallel derivatives appear in $\underset{\sim}{\mathbf{v}} \cdot \nabla \underset{\sim}{\mathbb{v}_{\|}} \cdot$ Finally, since $\underset{\sim}{\mathbb{B}_{1}}=0$, only $\mathrm{J}_{1} \times{\underset{\sim}{\mathrm{B}}}_{\|}$appears, and $\mathrm{J}_{1}$ is computed using only derivatives parallel to the wall. The resulting equation is

$$
\rho \frac{\partial \underline{v}_{\|}^{\prime}}{\partial t}=-{\underset{\sim}{\|}}_{\|} \cdot \nabla \underset{\sim \|}{v_{\|}}+\underset{\sim}{J_{1}} \times{\underset{\sim}{B}}_{\|}
$$

and every term is known at the wall. It is surprising that a current density must pass into the wall in order to drive any velocity there. 
For another example, consider the equation of continulty

$$
\frac{\partial \rho}{\partial t}=-\nabla_{\|} \cdot\left(\rho_{\sim \|}\right)-\nabla_{1} \cdot\left(\rho_{\sim 1}\right)
$$

If we use a linear extrapolation of $\rho_{\sim}$ through zero at the wall, we determine $\nabla_{\perp} \cdot\left(\rho{\underset{\sim}{1}}_{1}\right)$. The other derivatives involve only quantities known at the wall.

In the same way, ${\underset{\sim}{\|}}_{1}$ is advanced on the wall using a linear extrapolation of $\underset{\sim}{E_{\|}}$through zero at the wall.

The essential 1dea of marching boundary conditions is to rearrange the equations analytically until only those quantities which are zero at the wall appear under perpendicular derivatives.

Another special feature of the difference scheme concerns the initial and final timesteps. A time-centered leapfrog scheme gives a convergence error of order $(\Delta t)^{2}$. However, in order to start the time integration with all quantities given at the same timestep, or to stop the integration leaving all the data at the same timestep, a noncentered timestep is required on at least one of the two leapfrog grids. We have found that if the first timestep is $\eta \Delta t$, the second $(1+\eta) \Delta t$, and all subsequent timesteps are $2 \Delta t$, the choice of $n=1 / \sqrt{2}$ distributes the error evenly between the two grids. The derivation follows by examining the second-order term in the noncentral Taylor series expansion

$$
f(t+a)=f(t)+a f^{\prime}(t+b)+\left(\frac{1}{2} a^{2}-a b\right) f^{\prime}-(t)+\ldots
$$

which may be used to evaluate. $\partial \mathrm{f} / \partial \mathrm{t}$ with various combinations of noncentered finite differences.

A variety of methods have been used to verify the results of the 3-D nonlinear evolution code presented here: convergence tests; conservation of mass, magnetic flux, and energy; repeated time reversal; continuity of growth rate and symmetry; running single and double precision; and checking linear growth rates against other computations. 
One example of a convergence test is illustrated in Fig. 10, for an $\mathbf{m}=1$ instability corresponding to Case II. As the number of grid points is increased from $12 \times 12 \times 8$ to $27 \times 27 \times 16$, for example, both the time evolution and profiles of the variables exhibit the same behavior. By checking a number of cases, it has been found that an $18 \times 18 \times 12$ grid is adequate for most $\mathrm{m}=1$ instabilities at high beta. A $27 \times 27 \times 16$ grid was used in all the results presented in Section 5 . Note that these numbers include ghost points around the poloidal cross section, so this grid corresponds to $24 \times 24 \times 16$ intervals within the domain.

The difference scheme conserves magnetic flux and mass identically. Local conservation of magnetic flux $(\nabla \cdot B=0)$ follows from Faraday's law provided finite differences in orthogonal directions commute, which is true for the spatial difference scheme used here. Due to computer round off error, it is observed that $\nabla \cdot \underset{\sim}{B}$ wanders from zero by about one part in $10^{4}$ during the computation.

Conservation of energy is a useful diagnostic since energy is not conserved exactly by the finite difference equations, as explained in Section 2. Referring to Figs. 7,8, and 9, the change in total energy is observed to be about $10 \%$ or less of the kinetic energy, growing at the same rate until there is a sudden reversal at the end, and is orders of magnitude smaller than the total energy, Different forms of the pressure equation have been used with no apparent change in the results.

A sudden time reversal at the end of a computer run produces a remarkable result as the instability retraces its steps for a length of time which depends upon the grid size. If time is then reversed again, the instability evolves to the same final stage. These results will be reported in more detail elsewhere by $H$. R. Hicks.

The continuity of growth rate between the linear and nonlinear computations is illustrated in Fig. 10. This is routinely checked for all runs. The kinetic energy and other variables are observed to grow at correspondingly correct rates. Also, all the spatial symmetries of the linear eigenfunctions are preserved during the nonlinear computation. 
No difference is observed when the computation is carried out in double precision (using an 8-byte word as opposed to the usual 4-byte word length on the IBM $360 / 91$ computer). A nominal seven decimal digits of accuracy appears to be adequate.

More tests and diagnostics of the computation are in progress and will be reported in future papers. On the basis of tests made to date, we have considerable confidence in the results presented here. 


\section{CONCLUSIONS}

On the basis of computer simulation, it is observed that internal ideal MHD instabilities in a straight cylinder are characterized by pairs of velocity vortex cells rolling of each other and helically twisting down the plasma column. Pressure, temperature, density, perturbed magnetic field, and perturbed current density convect around these essentially fixed velocity vortex cells. Compression and other effects are also observed. Spatial localization is the most important quality affecting the nonlinear evolution. After a significant amount. of convection, the velocity vortex cells stop growing and begin to wander.

A wide variety of different conditions has been investigated with the same model, including the effects of different profiles, elongated cross sections and wavelengths, $\beta_{p}$ different from one, and toroidicity. These will be reported in future papers. Qualitative aspects of the results shown here hold for the wide range of cases studied to date. In addition, a number of projects are under way to include transport and source terms, different boundary conditions, and improved diagnostics. Initial value MHD codes will continue to provide a very flexible tool for studying large-scale phenomena. 


\section{ACKNOWLEDGMENTS}

We wish to thank R. A. Dory for the discussions and the interest he has shown in this work. We also wish to acknowledge programming contributions by J. F. Roberts. 


\section{THIS PAGE}

\section{WAS INTENTIONALLY LEFT BLANK}




\section{REFERENCES}

[1] BATEMAN, G., SCHNEIDER, W., GROSSMANN, W., Report IPP 1/145 (Apri1 1974); Nuc1. Fusion 14 (1974) 669-683.

[2] SCHNEIDER, W., BATEMAN, G., in Plasma Physics and Controlled Nuclear Fusion (Proc. 5th Int. Conf. Tokyo, 1974) IAEA-CN-33/A 12-1.

[3] WOOTEN, J. W., HICKS, H. R., BATEMAN, G., DORY, R. A., Preliminary Results of the 3-D Nonlinear Ideal MHD Code, Oak Ridge National Laboratory, ORNL/TM-4784 (November 1974).

[4] HICKS, H. R., WOOTEN, J. W., BATEMAN, G., Linear-MHD Instability Code, Bull. Am. Phys. Soc. 19 (19.74) 969.

[5] WOOTEN, 'J.'W., HICKS, H. R., A Fast Symbolic MHD Instability Code, Bu11. Am. Phys. Soc. 19 (1974) 968.

[6] ' BATEMAN, G.; DORY, R. A., HiCKS, H. R., WOOTEN, J. W., 3-D Nonlinear Evolution of Internal MHD Instabilities, Sherwood Theory Meeting (April 1975).

[7] WOOTEN, J.W., HICKS, H. R.; Preliminary Description of the Linear and Nonlinear Ideal MHD Codes, Report UCC-CSD-INF-21 (May 1975).

[8] HICKS, H. R., WOOTEN, J. W., DORY, R. A., BATEMAN, G. . Computation of MHT Instabilities in Tokamaks, in Numerical Simulation of Plasmas (Proc. 7th Conf. New York, June 1975).

[9] BATEMAN, G., HICKS, H. R., WOOTEN, J. W., DORY, R.. A., Nonlinear Evolution of MHD Instabilities in Controlled Fusion and Plasma Physics (presented at the 7 th European Conf., Lausanne, September 1975).

[10] BATEMAN, G., HICKS, H. R., WOOTEN, J. W., Nonlinear MHD Instabilities in Elongated Cylinders, in Pulsed High Beta Plasmas (3rd Topical Conf., Culham, England, September 1975).

[11] BATEMAN, G., HICKS, H. R., WOOTEN, J. W., Effect of Profile and. Cross Section on the Nonlinear Evolution of MHD Instabilities, Bu11. Am. Phys. Soc. 20 (1975) 1256.

[12] SWIERZBINSKI, J. S., ROBERTS, J. F., BATEMAN, G., HICKS, H. R., WOOTEN, J. W., Nonlinear Evolution of MHD Instabilities in Axisymmetric Toroids, Bul1. Am. Phys. Soc. 20 (1975) 1264. 
[13] FURTH, H. P., Nucl. Fusion 15 (1975) 487-534.

[14] TFR Group (presented by D. Launois), in Controlled Fusion and Plasma Physics (Proc. 7th European Conf. Lausanne, 1975) 2, 1-13.

[15] MIRNOV, S. V., SEMENOV, I. B., Sov. Phys.-JETP 33 (1971) 1134-1137; Atomnaya Energiya 30 (1971) 20-27.

[16] HOSEA, J. C., BOBELDIJK, C., GROVE, D. J., in Plasma Physics and Controlled Nuclear Fusion Research (Proc. 4th Intl. Conf.) 2 (1971) 425-440.

[17] BOL, K., et a1., in Plasma Physics and Control.1ed Nur.1par Fusinn Research (Proc.Int. Conf. Tokyo, 1974) 1 , 83-97.

[18] VON GOELER, S., STODIEK, W., SAUTHOFF, N., Phys. Rev. Lett. 33 (1974) 1201; VON GOELER, S., in Controlled Fusion and Plasma Physics (Proc. European Conf. Lausanne, 1975) 2, 71-80.

[19] ORMAK group (presented by LYON, J. F.), in Controlled Fusion and Plasma Physics (Proc. 7th European Conf. Lausanne, 1975) 2, 24-37.

[20] KARGER, F., et a1., in Plasma Physics and Controlled Nuclear Fusion Research (Proc. 5th Int. Conf. Tokyo, 1974) 1, 207-213.

[21] WADDELL, B. V., et al., submitted to Nucl. Fusion.

[22] POTTER, D., Computational Physics, Wiley, 1973.

[23] RICHTMYER, R. D., MORTON, K. W., Difference Methods for InitialValue Problems, Interscience, Wiley, 1967.

[24] SHAFranov, V. D., Sov. Phys.-Tech. Phys. 15 (1970) 175-183.

[25] BATEMAN, G., submitted to Nucl. Fusion.

[26] ABRAMOWITZ, M., SEGUN, I.A., Handbook of Mathematical Functions, Dover, P. 590 (1964).

[27] GOEDBlOED, J. P., SAKANAKA, P. H., Phys. Fluids 17 (1974) 908-929.

[28] GROSSMANN, W., ORTOLANI, S., Max Planck Institut für Plasmaphysik Report IPP 1/132 (April 1973). 


\section{F1gure Captions}

F1g. 1. Geometry and coordinate systems for the straight rectangular cylinder with sides $2 a \times 2 b \times \lambda$. The cylinder has rigid, perfectly conducting walls at the sides and periodic conditions at the ends.

Fig. 2. The q-value as a function of $x$ along the midplane of a square cylinder for the equilibrium specified by Eqs. (3.12) and (3.13). The three curves correspond to central q-values of $1.6,0.95$, and 0.6 . The radius of the mode-rational surface $\left(r_{s}\right)$ is indicated in each case.

Fig. 3. Growth rate as a function of central q-value for $m=0,1$, 2 , and 3 modes of wavelengths 1,2 , and 3 in units of $2 \pi a$. Solid curves refer to a cylinder with square cross section and equilibrium specified by Eqs. (3.12) and (3.13). Dashed curves refer to a circular cylinder with parabolic current profile.

Fig. 4. Time sequences for a cross section of velocity, pressure, perturbed magnetic field, poloidal current density, rate of change of velocity $\nabla \cdot \mathrm{v}$, longitudinal current density and mass density for an $\mathrm{m}=1$ instability with $\mathrm{nq}_{\mathrm{c}}=0.95, \mathrm{ka}=1, \gamma=0.24$, Case $\mathrm{I}$ in Table $\mathrm{I}$, run 1087. Time is in units of the reference Alfien transit time.

Minimum and maximum values appear under each frame. See Appendix I for the interpretation of the graphs.

Fig. 5. Time sequences as in Fig. 4 for an $m=1$ instability with $\mathrm{nq}_{\mathrm{c}}=0.6, \mathrm{ka}=1, \gamma=0.535$, Case II in Table 1 , run 1088 .

Fig. 6. Time sequences as in Figs. 4 and 5 for an $m=2$ instability with $\mathrm{nq}_{\mathrm{c}}=1.6, \mathrm{ka}=1, \gamma=0.117$, Case III in Table 1 , runs 1086 and 1107. The small longitudinal components near the corners in the first frame of $\partial v / \partial t$ are a numerical artifact of unknown origin. 
Fig. 7. Maximum poloidal velocity, kinetic energy, and decrement in total energy as a function of time for an $m=1$ mode corresponding to Case I, run 1087. Total energy is 0.779 . Arrows point to instants in time illustrated in Fig. 4.

Fig. 8. Maximum poloidal velocity, kinetic energy, and energy decrement as a function of time for Case II, run 1088. Total energy is 1.20. Arrows point to instants in time illustrated in Fig. 5.

Fig. 9. Maximum poloidal velocity, kinetic energy, and energy decrement as a function of time for the $m=2$ mode, Case III, runs 1086 and 1107. Total energy is 0.598. Arrows indicate last two instants in time illustrated in Fig. 6; the first instant is off the graph to the left.

Fig. 10. Instantaneous growth rate of the central poloidal velocity, $v_{y}(t)$, as a function of time during the nonlinear evolution of an $m=1$ instability with $\mathrm{nq}_{c}=0.6$ corresponding to Case $\mathrm{I}$ in Section 5 . The results are shown for three different grids. Lines with arrows indicate results of time reversal. 


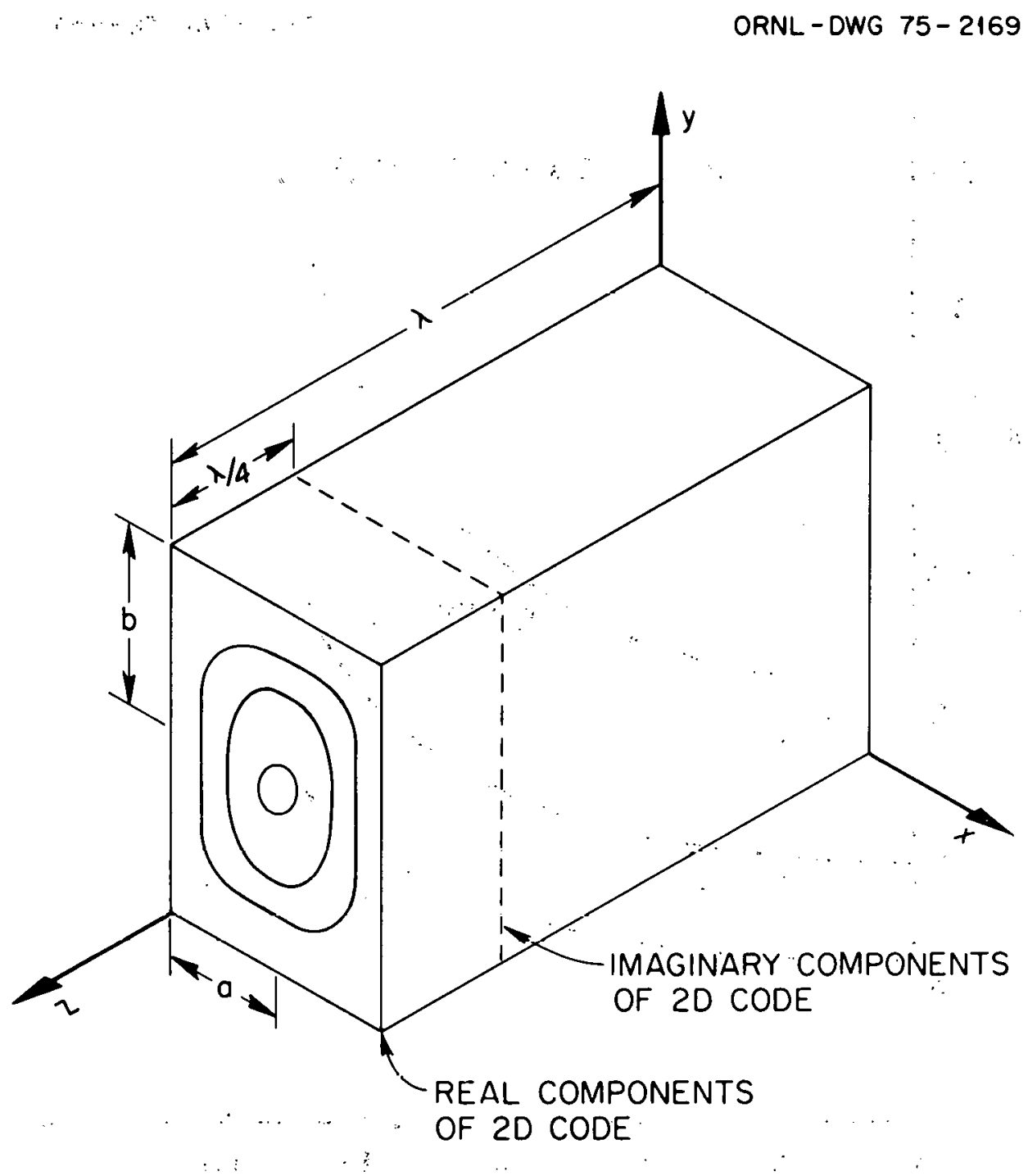

Fig. 1 


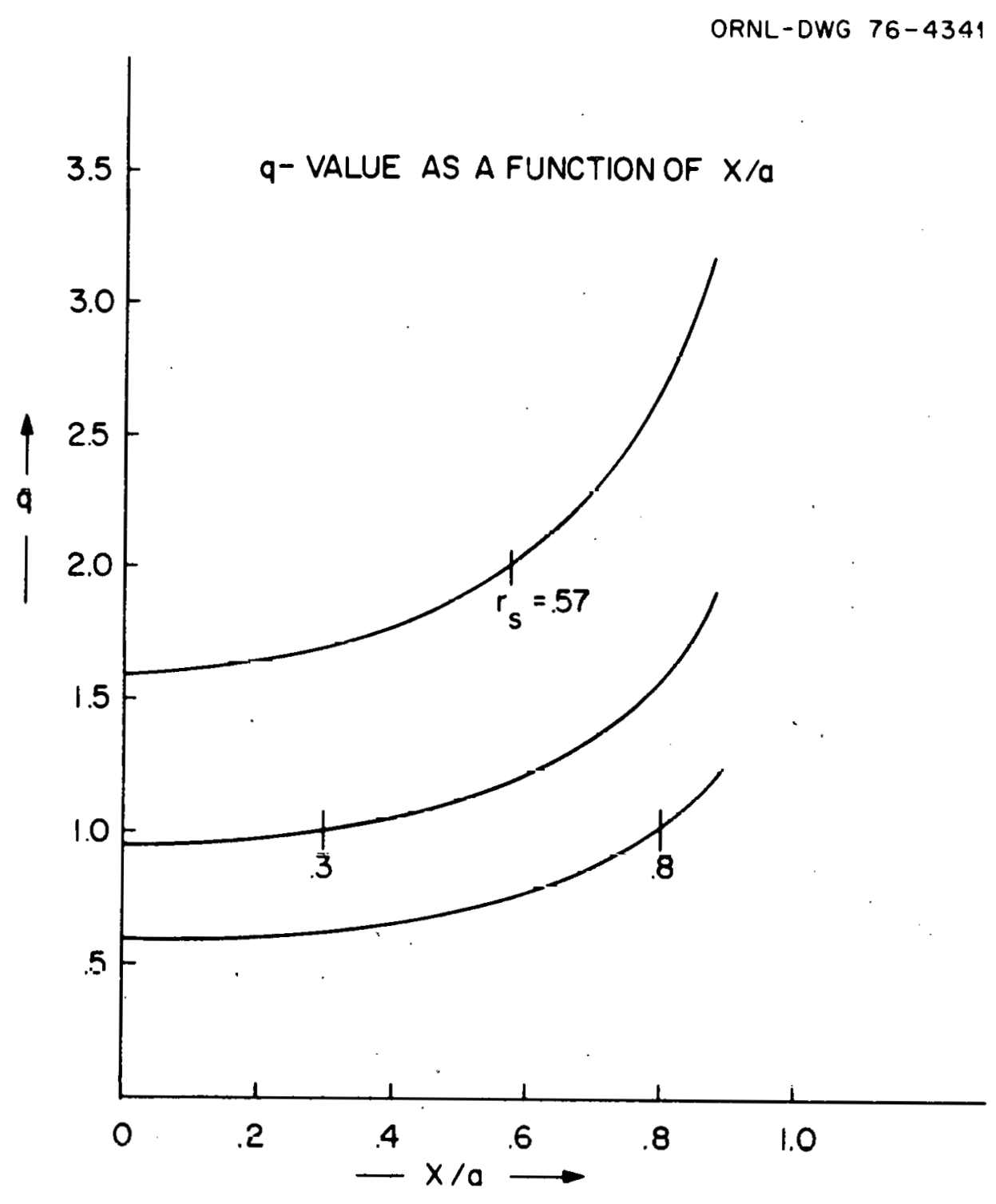

Fig. 2 
ORNL-DWG $76-4346$

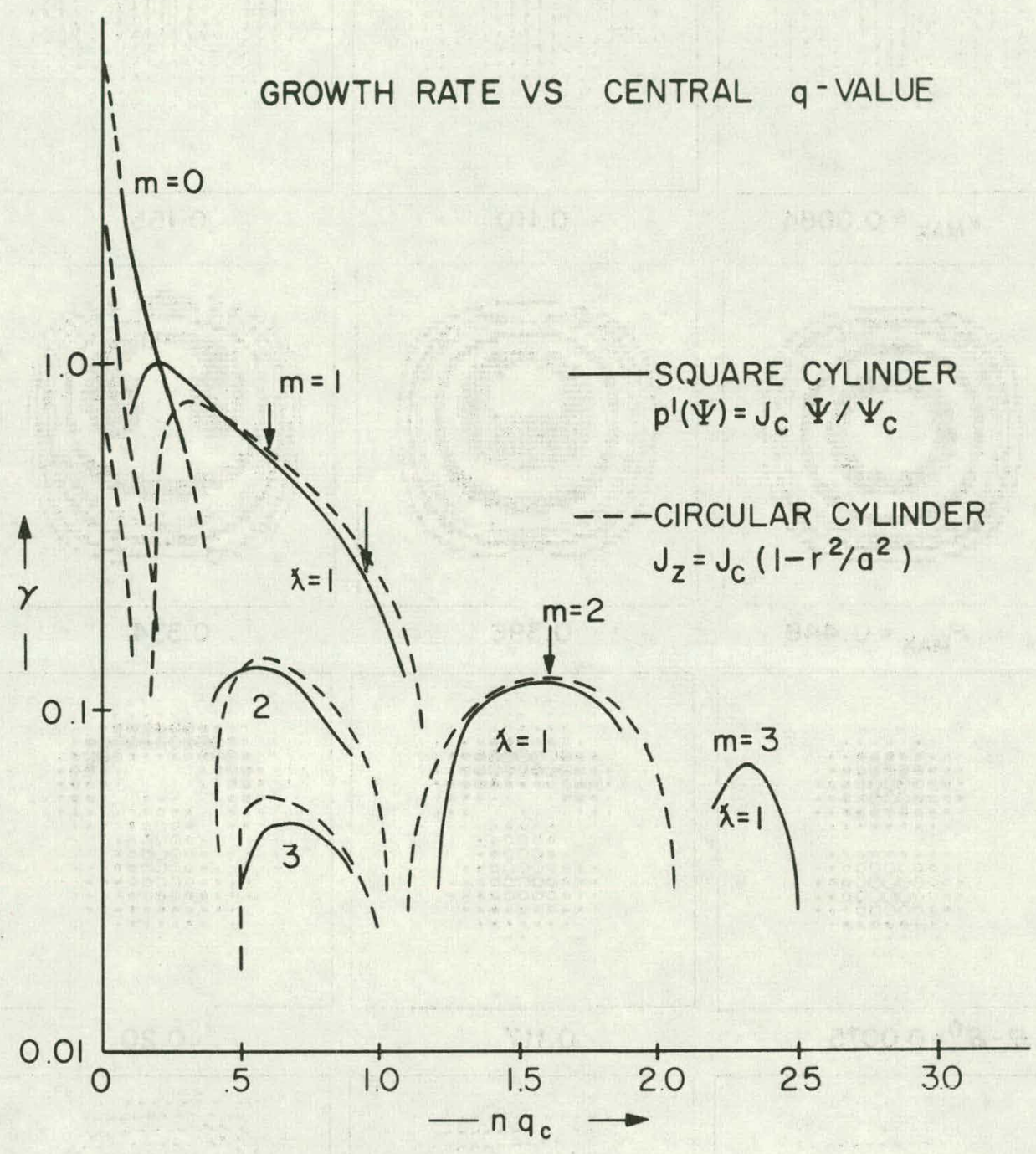

Fig. 3 

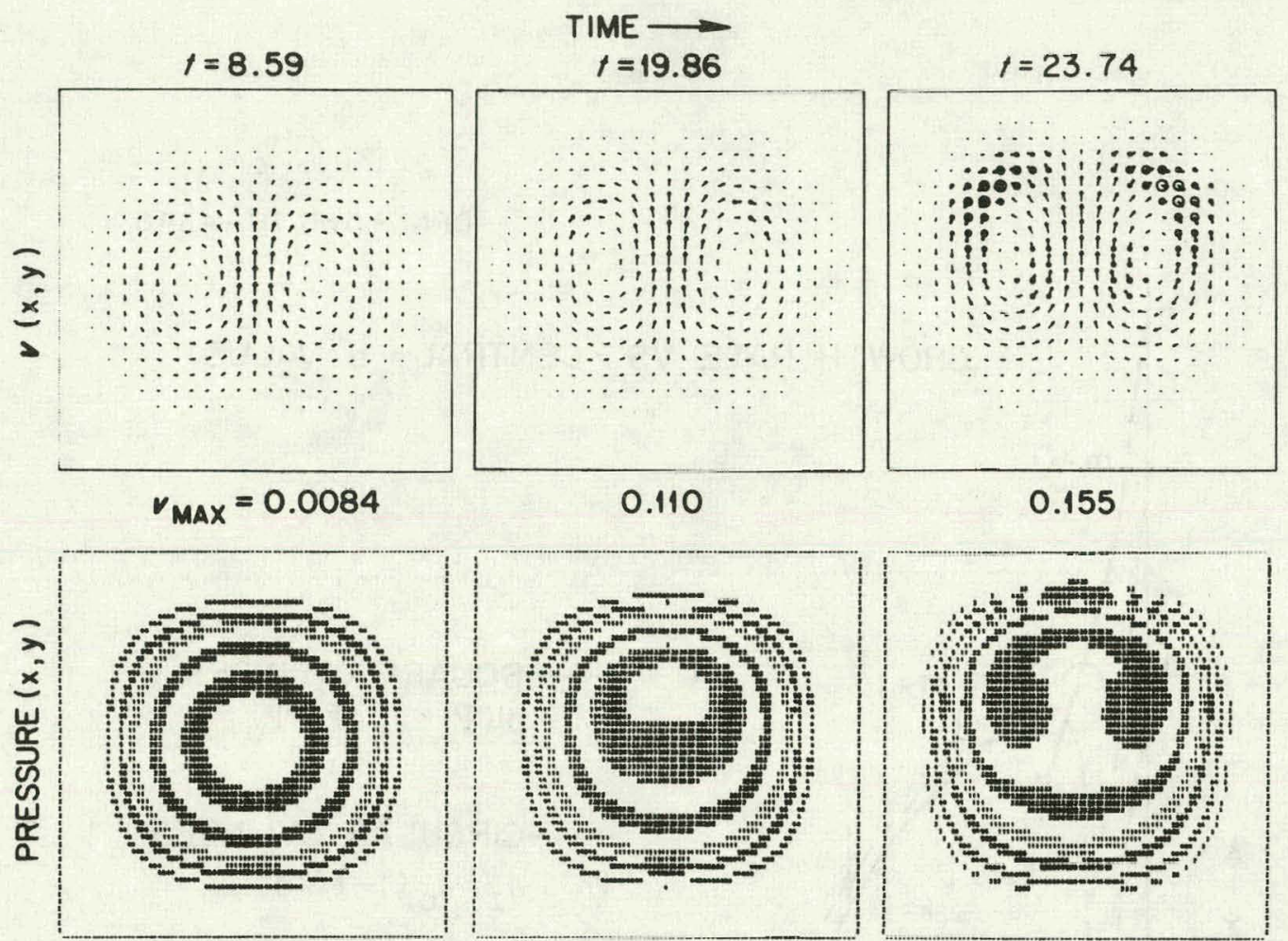

$$
P_{\text {MAX }}=0.448
$$

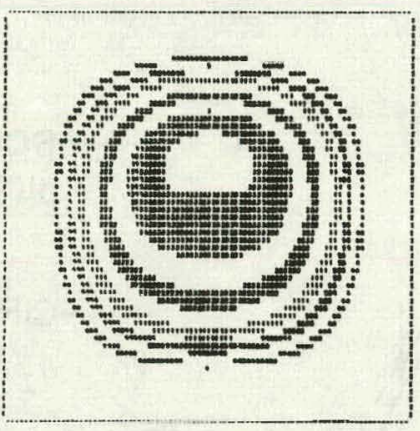

$$
0.396
$$
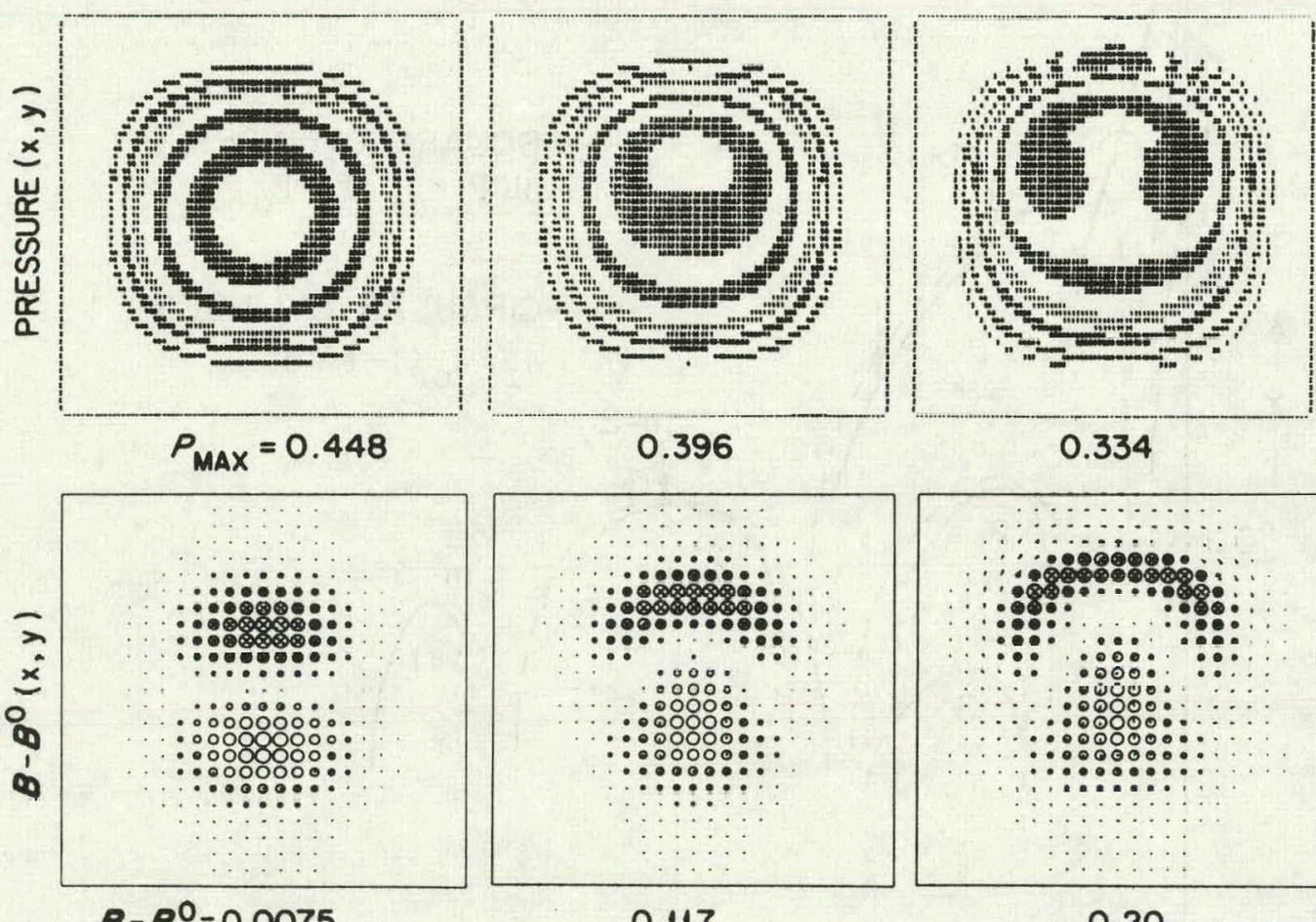

$$
0.117
$$
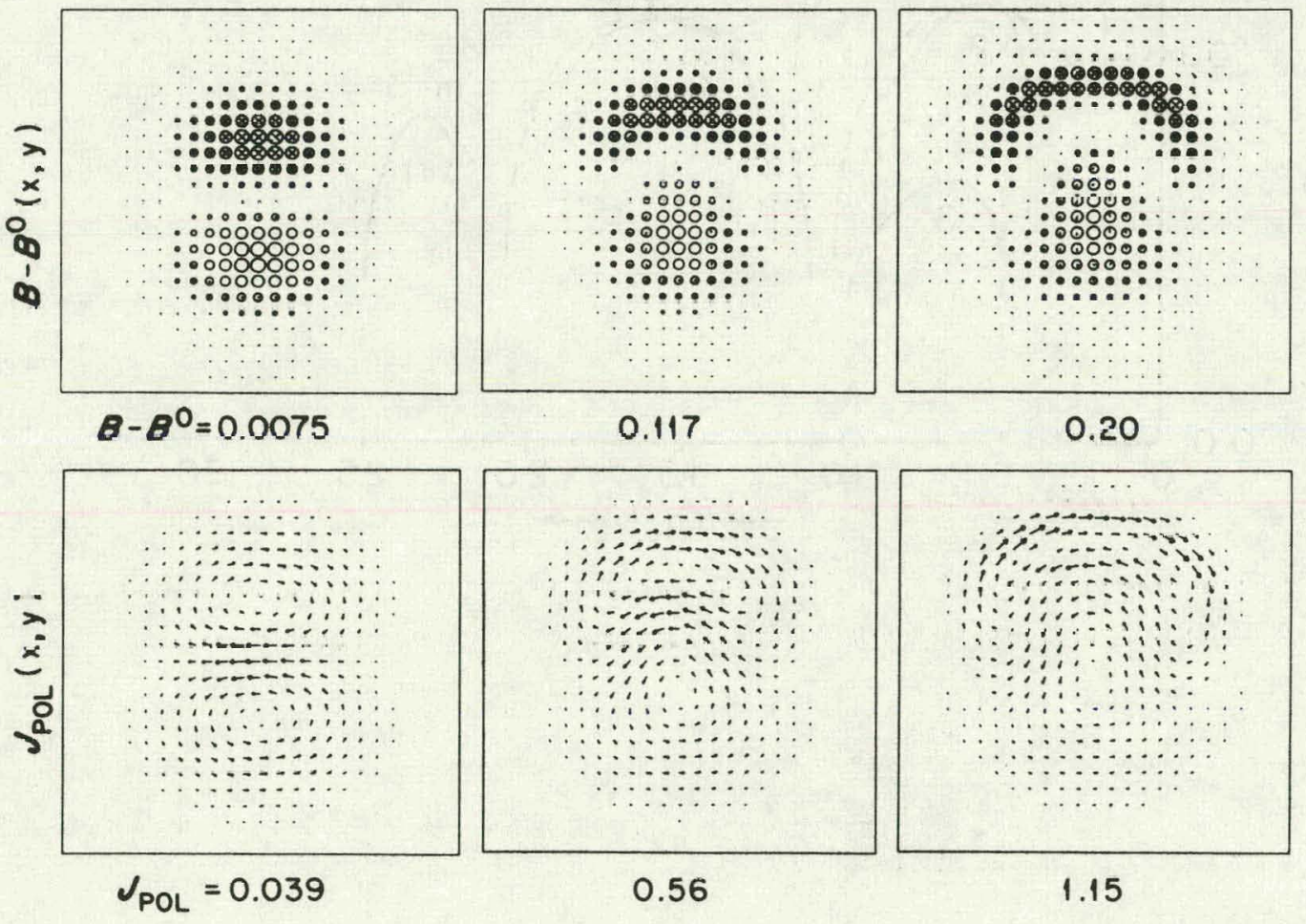

$$
\begin{gathered}
m=1 \quad n q_{c}=0.95 \quad k a=1 \quad \gamma=0.24 \quad \text { RUN } 1087 \\
\text { Fig. } 4 a
\end{gathered}
$$



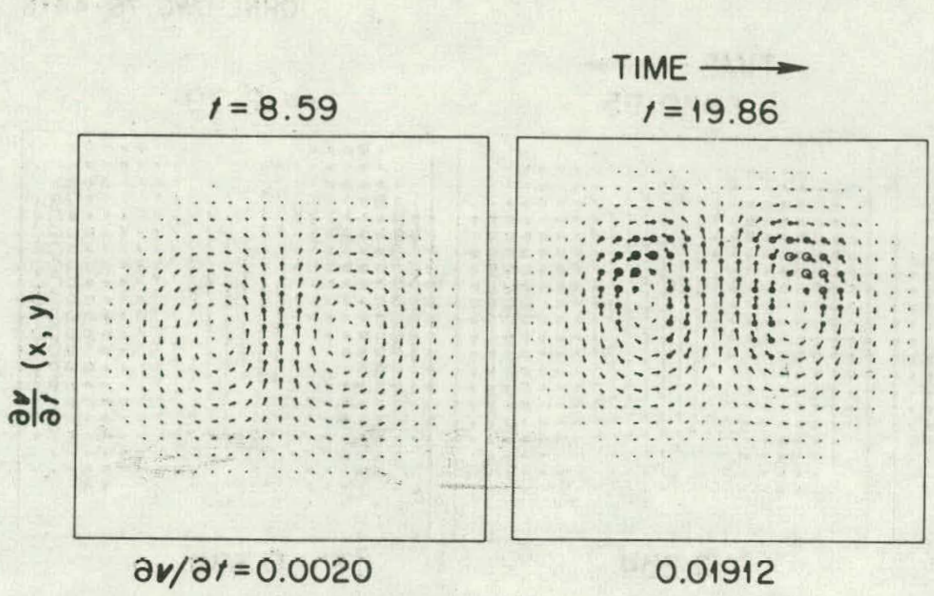

ORNL-DWG 76- 4427
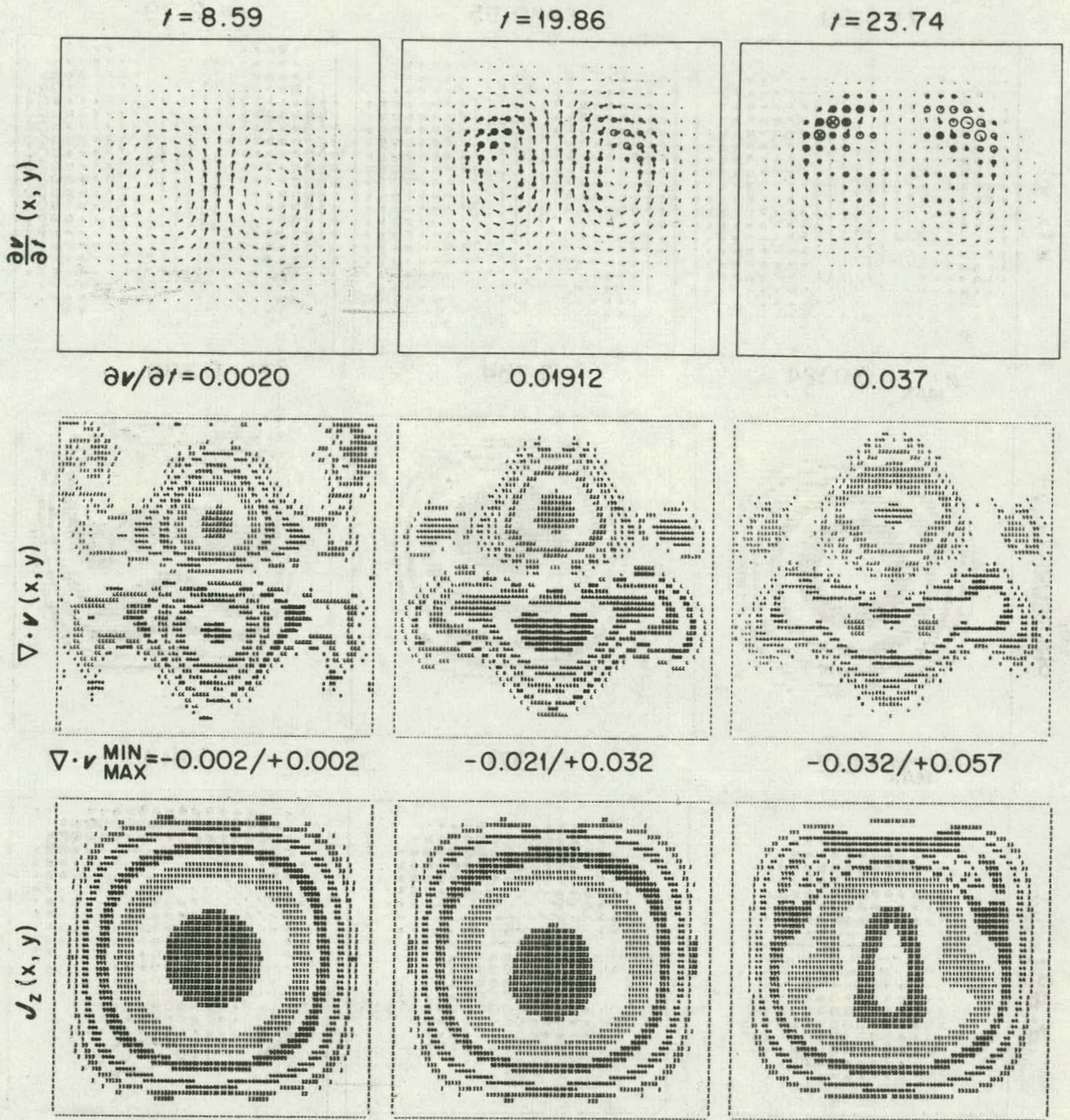

$$
J_{z}=2.1
$$

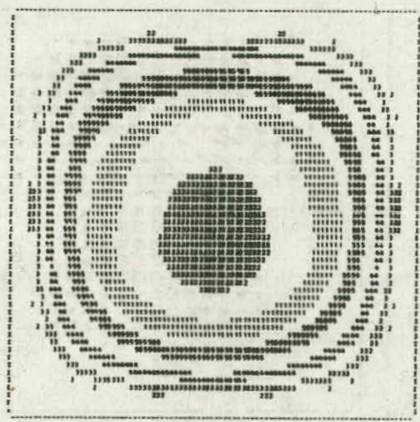

$$
2.215
$$
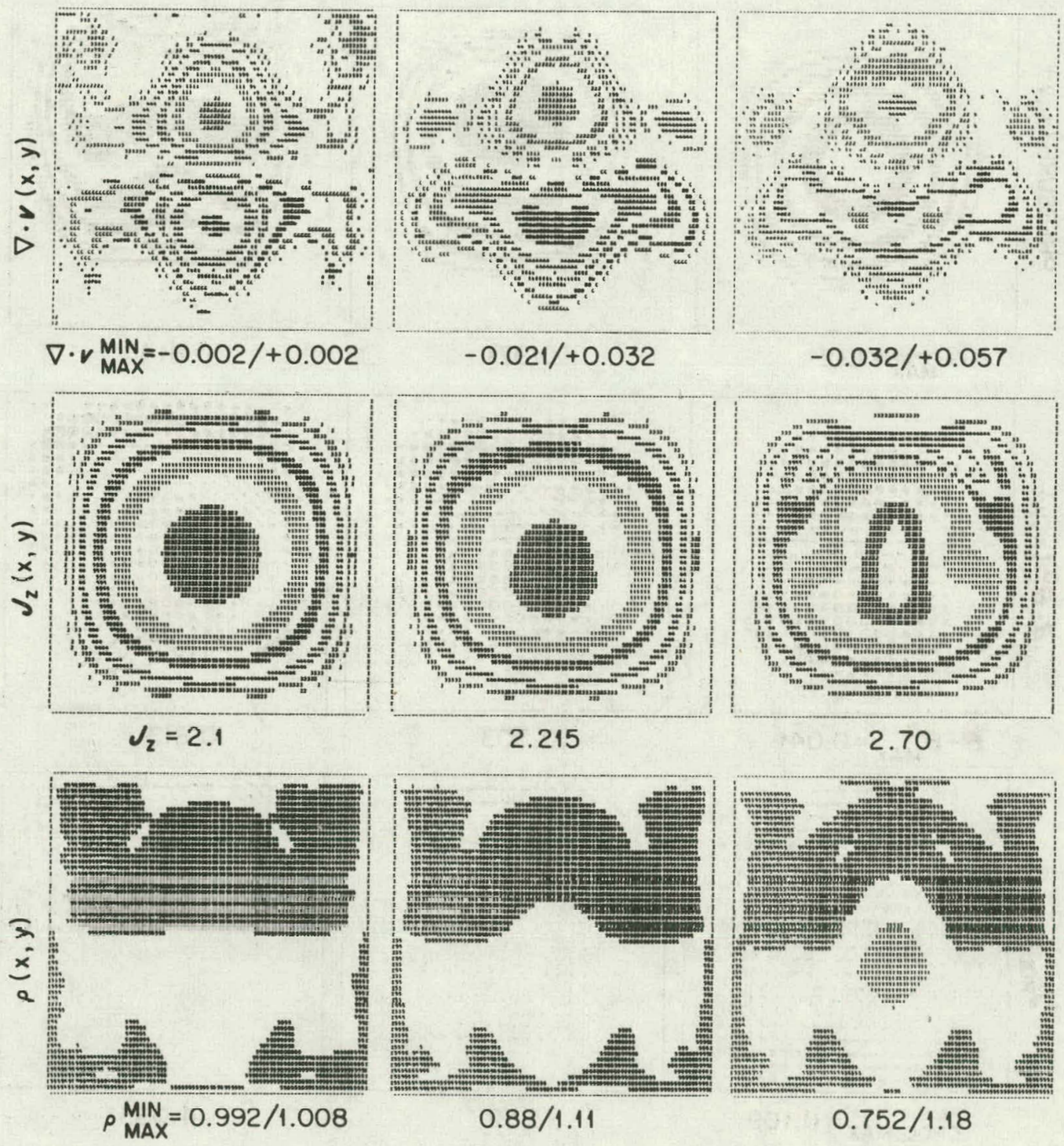

2.70

$m=1 \quad n q_{c}=0.95 \quad k a=$

$$
\gamma=0.24
$$

RUN 1087

Fig. 4b 


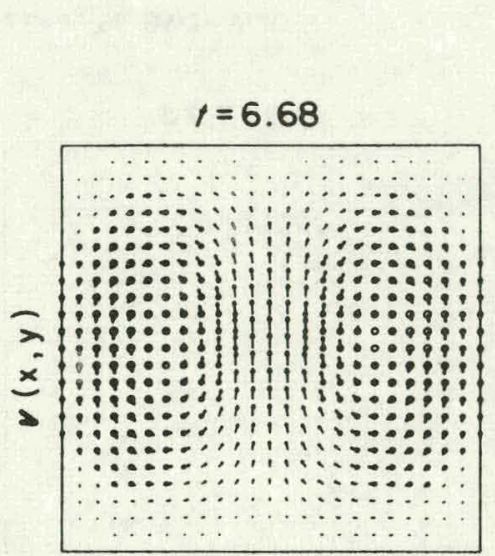

$$
v_{\text {MAX }}=0.0324
$$
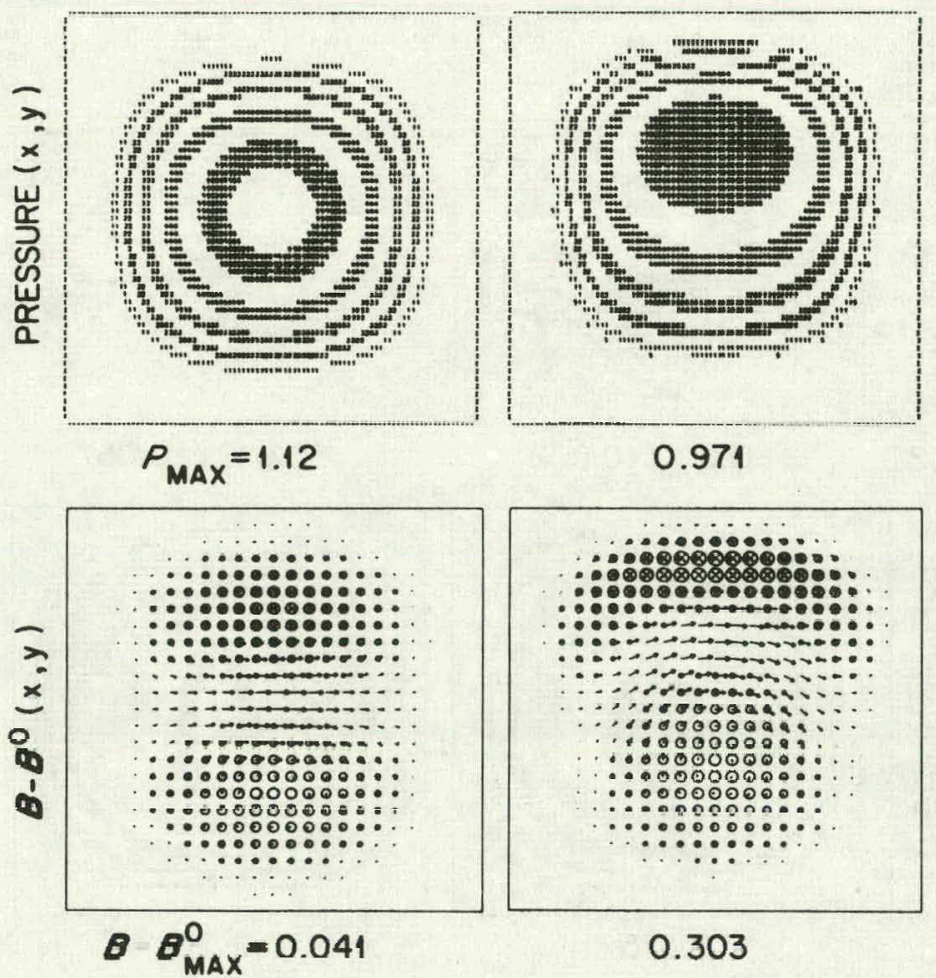

0.303
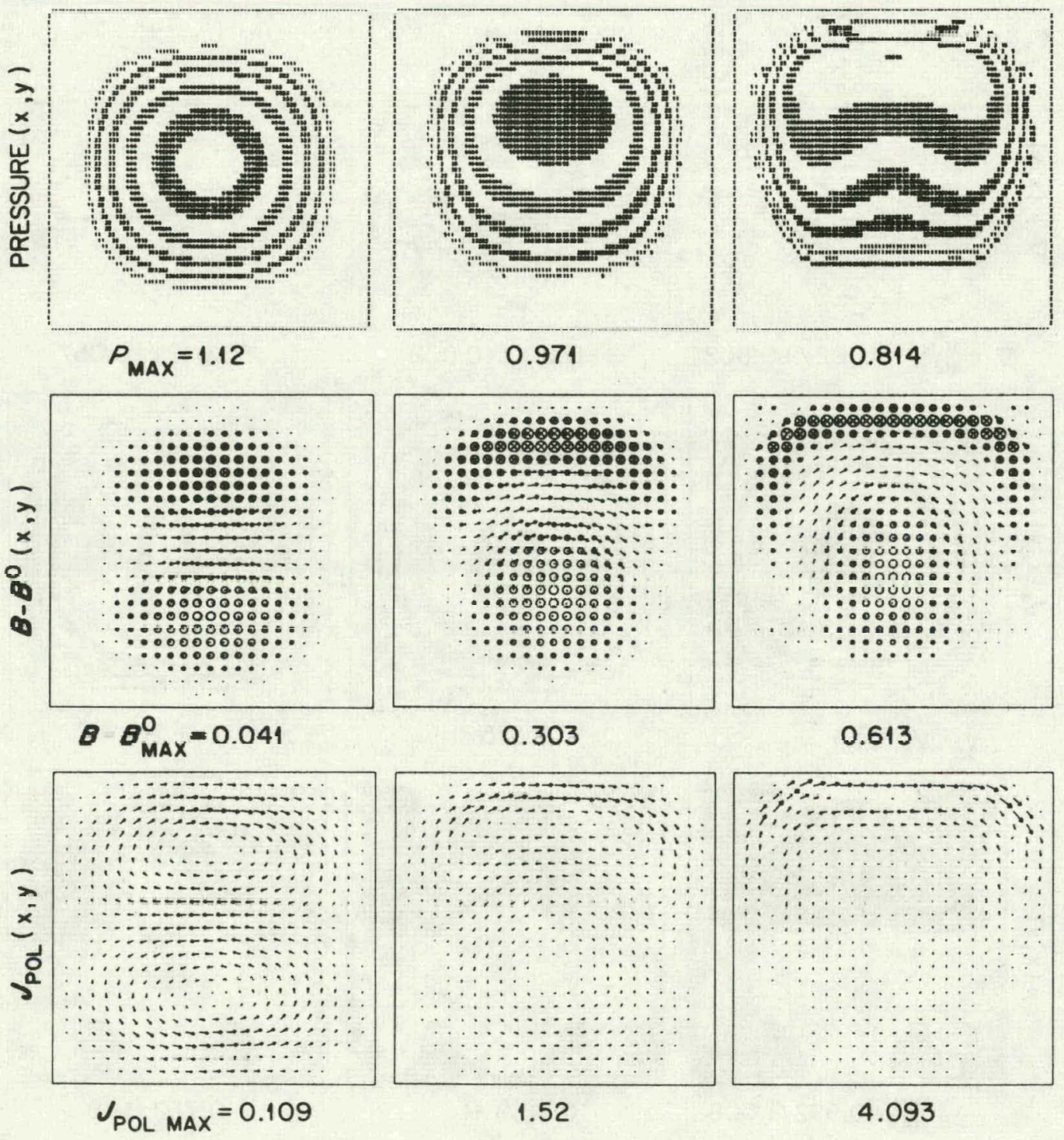

$m=1 \quad n q_{c}=0.6 \quad k a=1 \quad \gamma=0.535 \quad$ RUN 1088

Fig. $5 a$ 
ORNL-DWG $76-4430$
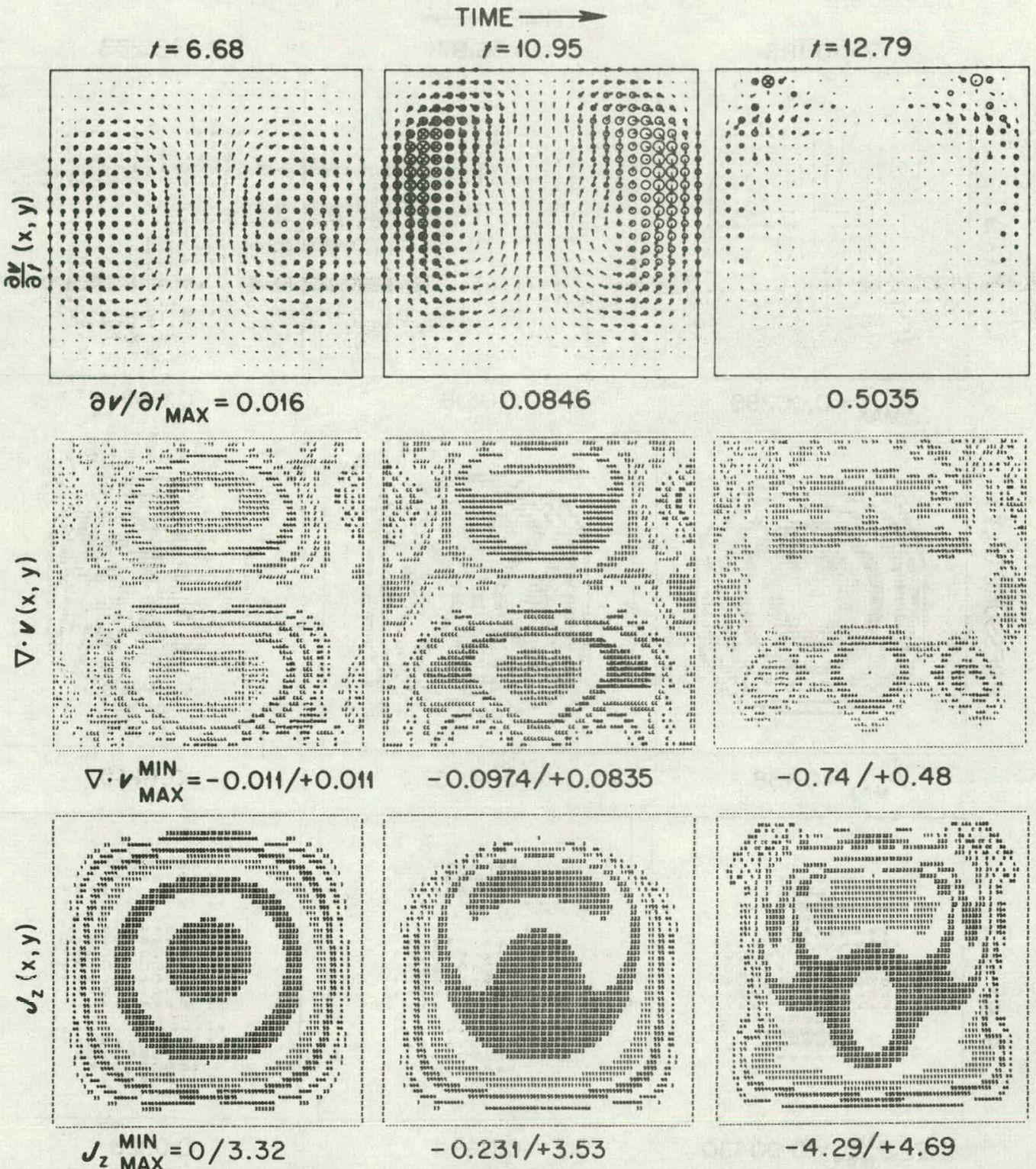

$-0.74 /+0.48$
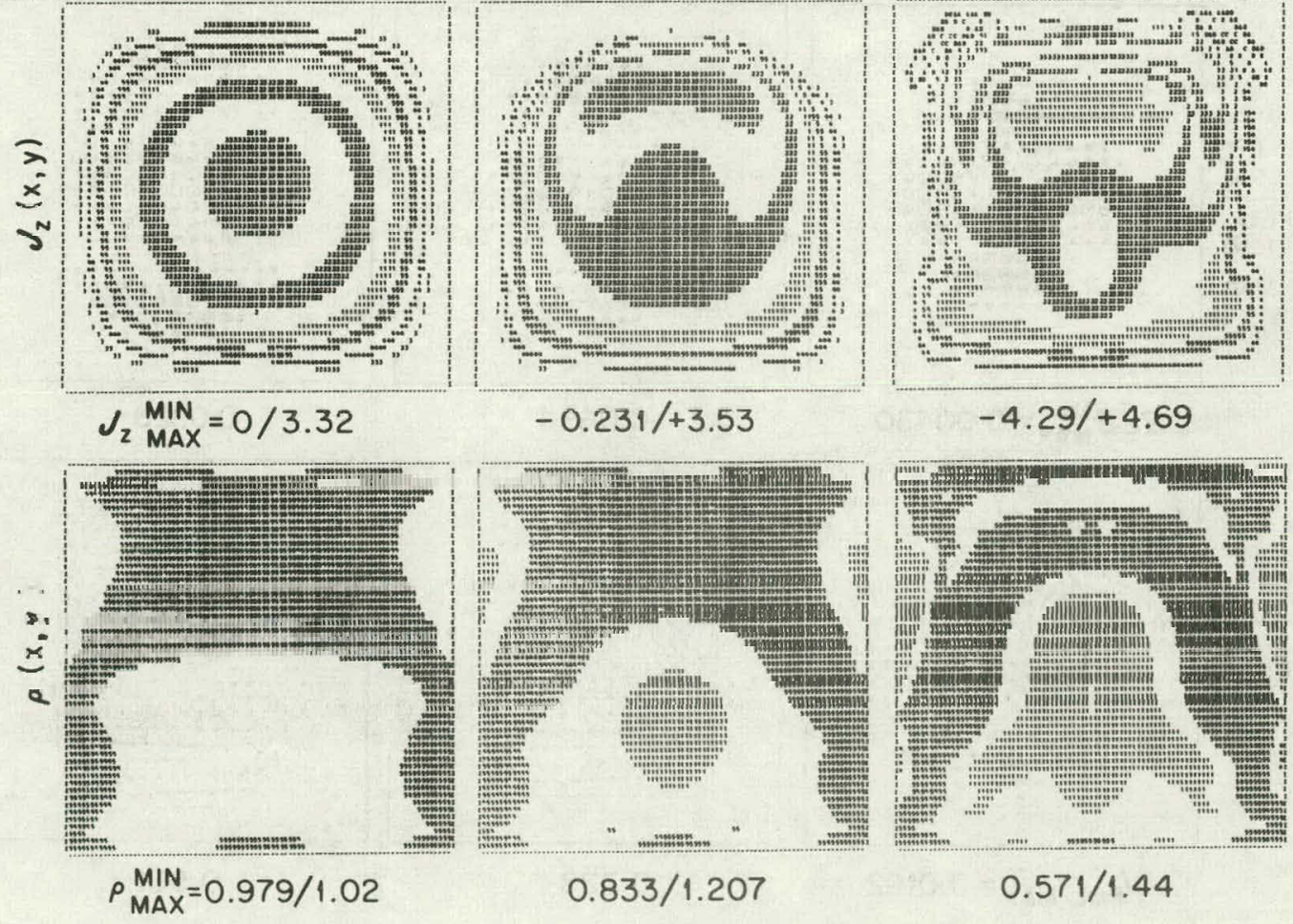

$m=1 \quad n q_{c}=0.6 \quad k a=1 \quad \gamma=0.535 \quad$ RUN 1088

\section{$0.571 / 1.44$}

Fig. 5b 

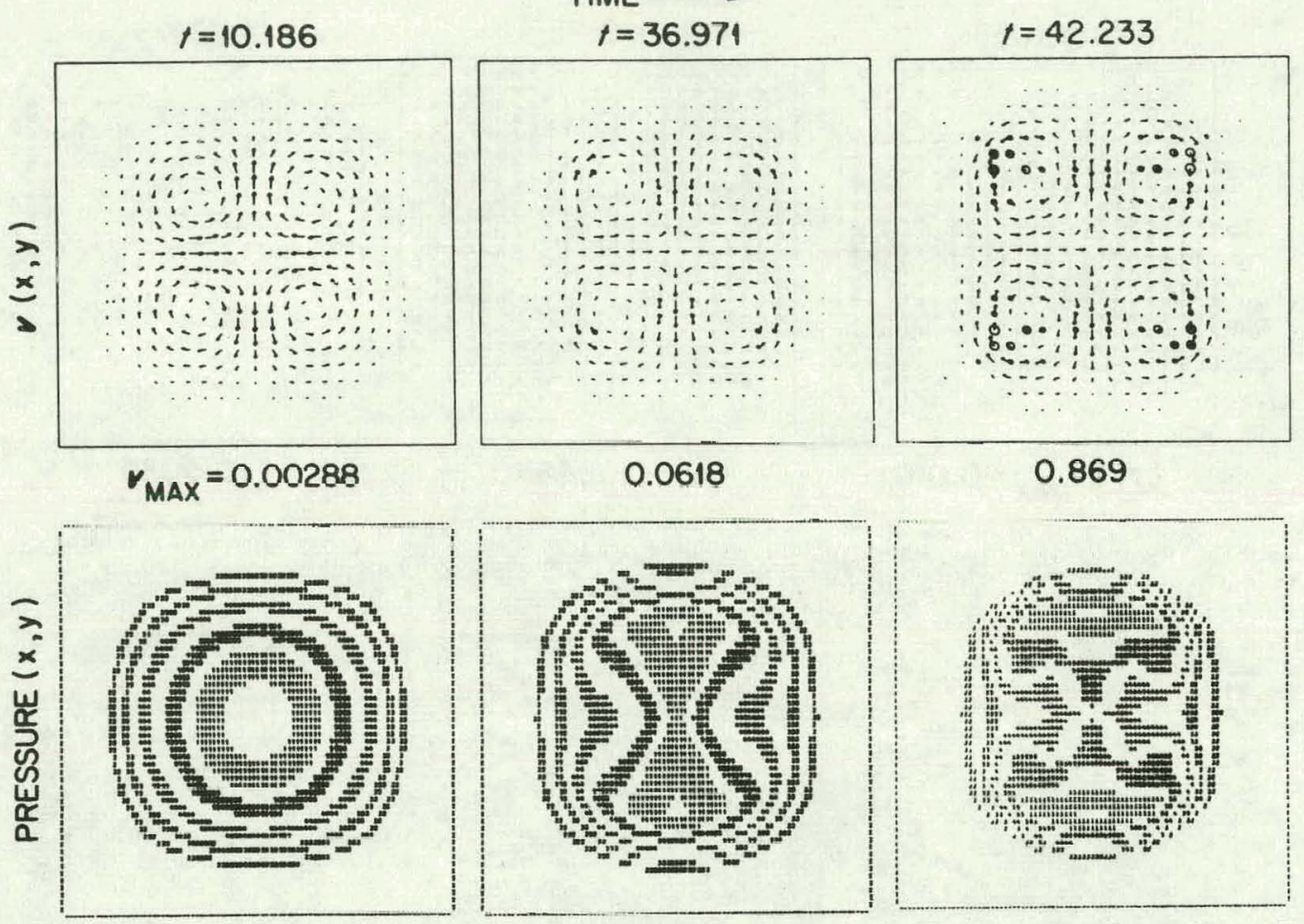

$$
P_{\text {MAX }}=0.158
$$
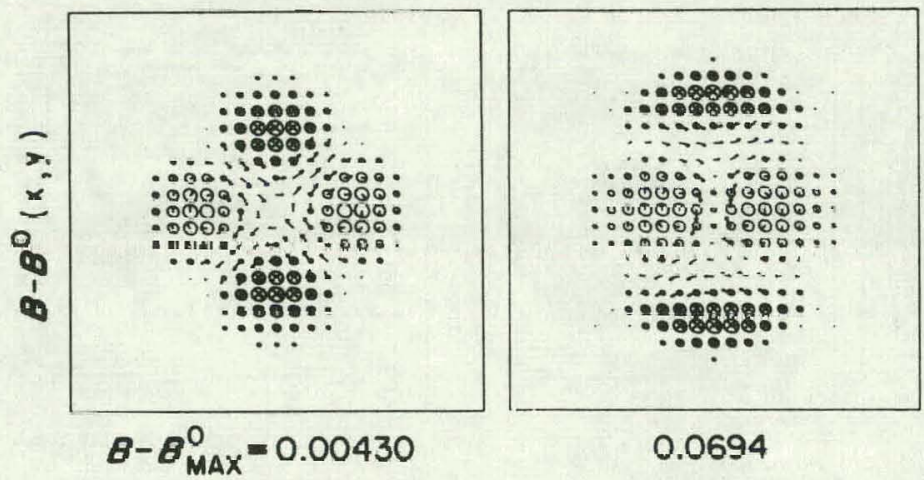

\subsection{4}

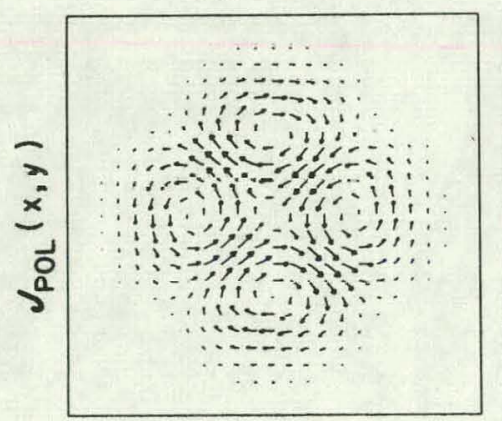

$J_{\text {POL MAX }}=0.0162$

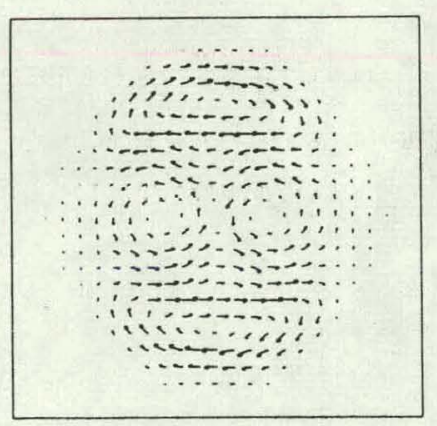

0.328

ORNL-DWG 76-4414

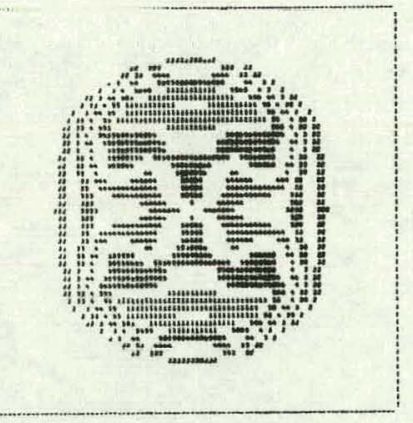

0.1445
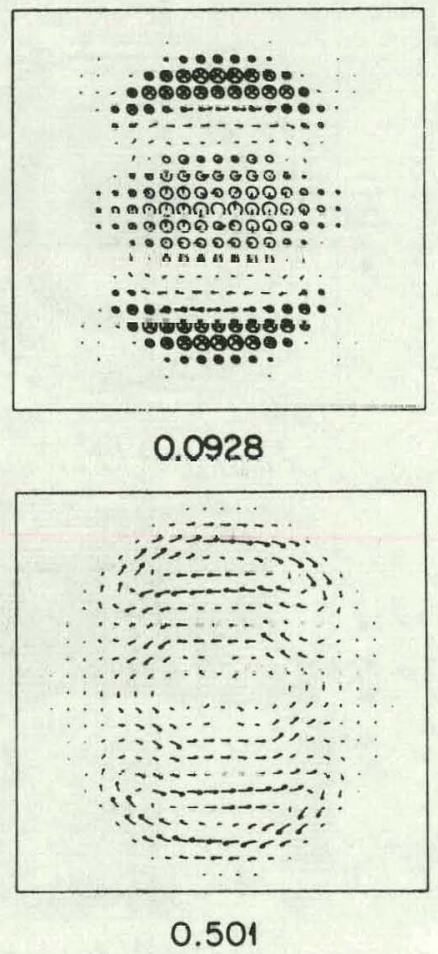

$m=2 \quad n a_{c}=1.6 \quad k a=1 \quad \gamma=0.117$ RUNS 1086 AND 1107

Fig. $6 a$ 
ORNL-DWG 76-4429
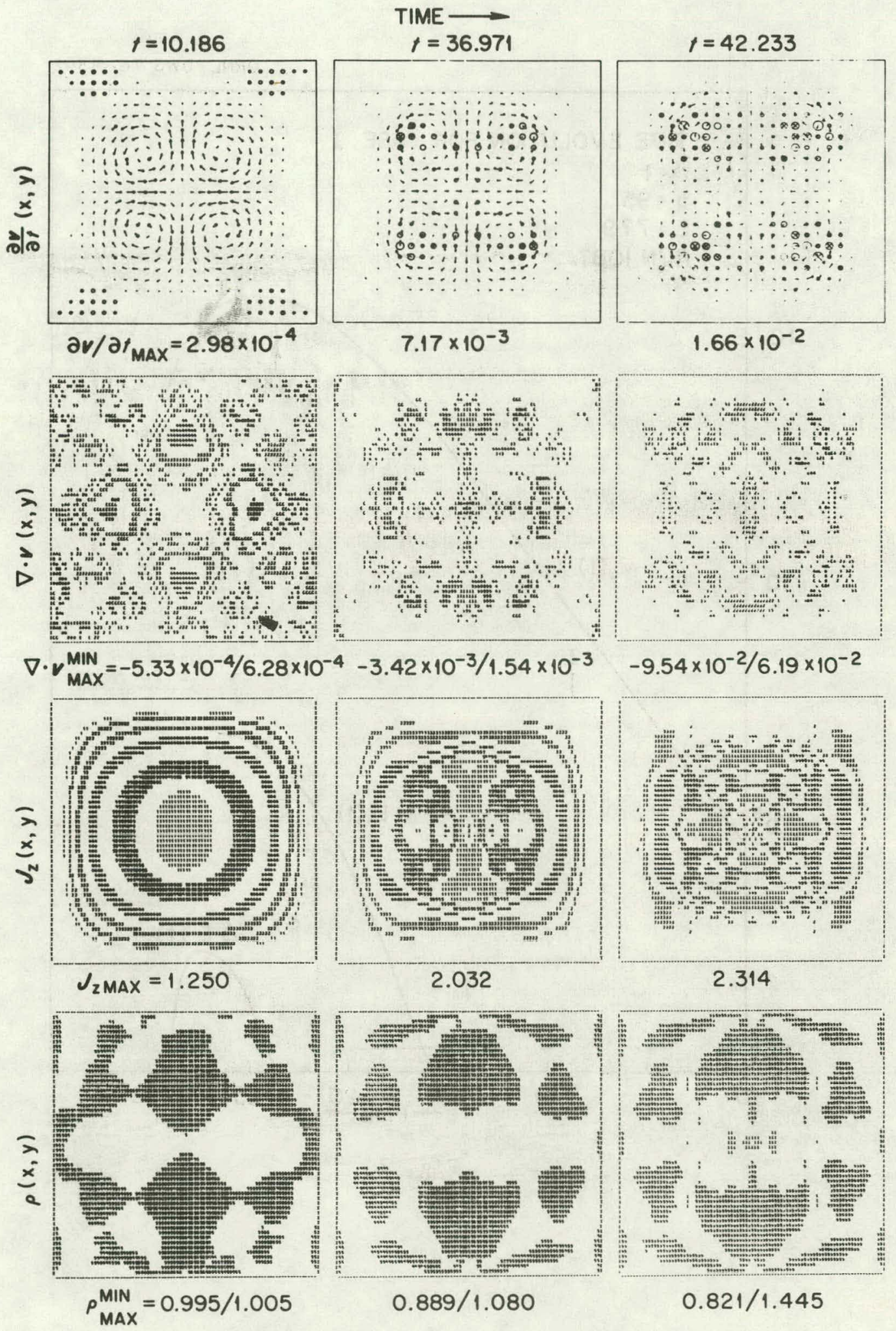

$m=2 \quad n q_{c}=1.60 \quad k n=1$

$\gamma=0.117 \quad$ RUNS 1086 AND 1107

Fig. 6b 


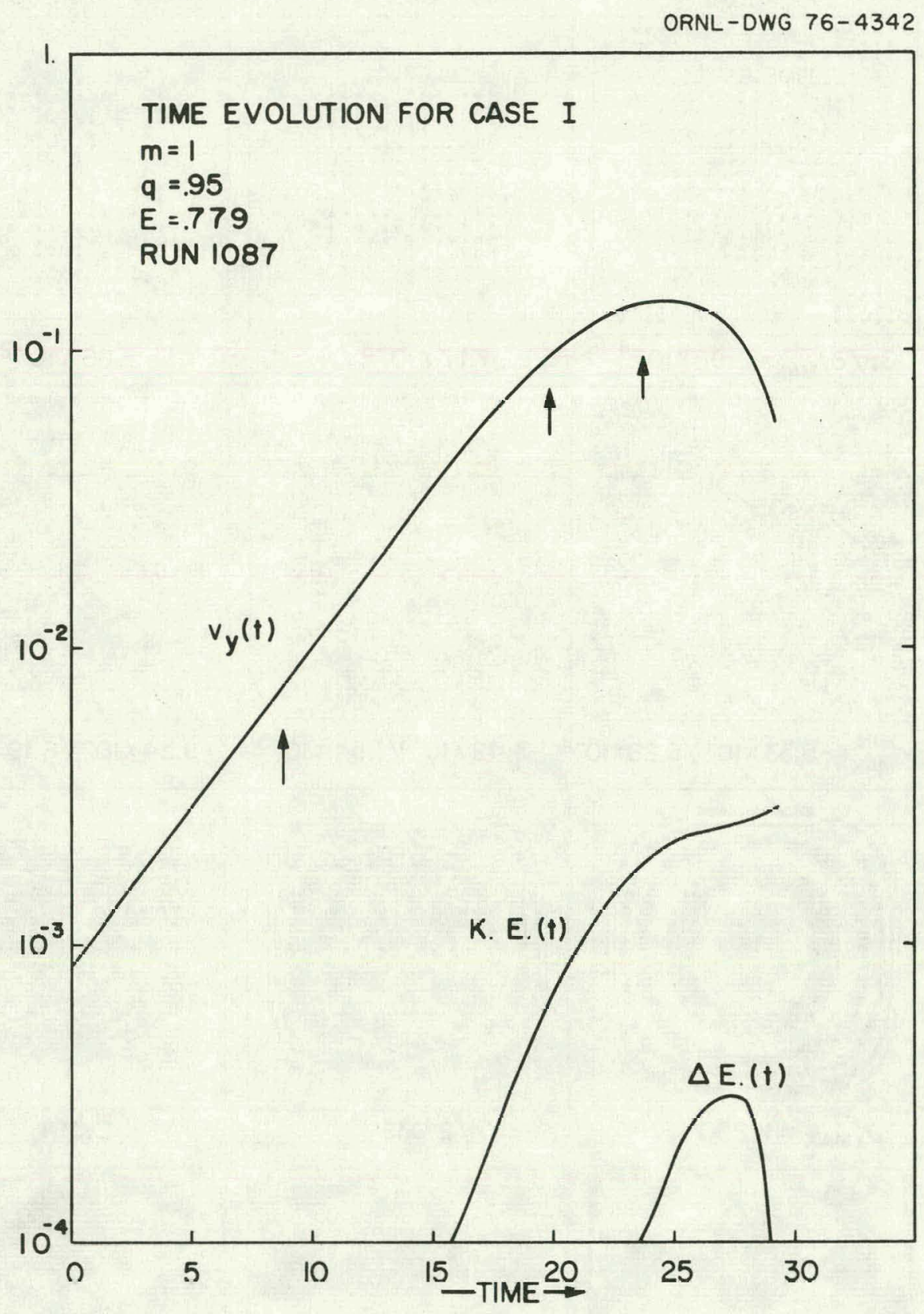

Fig. 7 


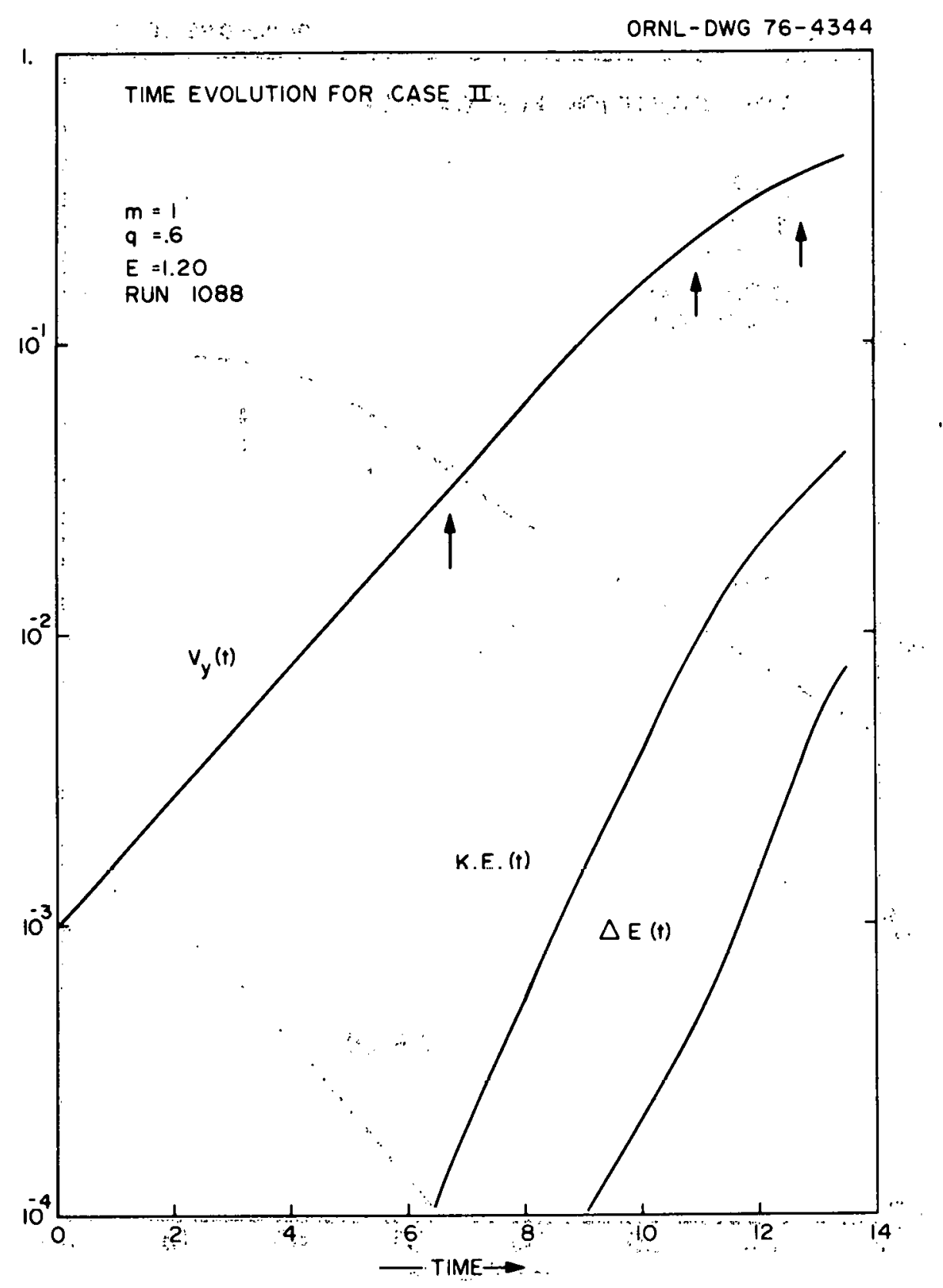

Fig . 8 
ORNL-DWG 76-4343

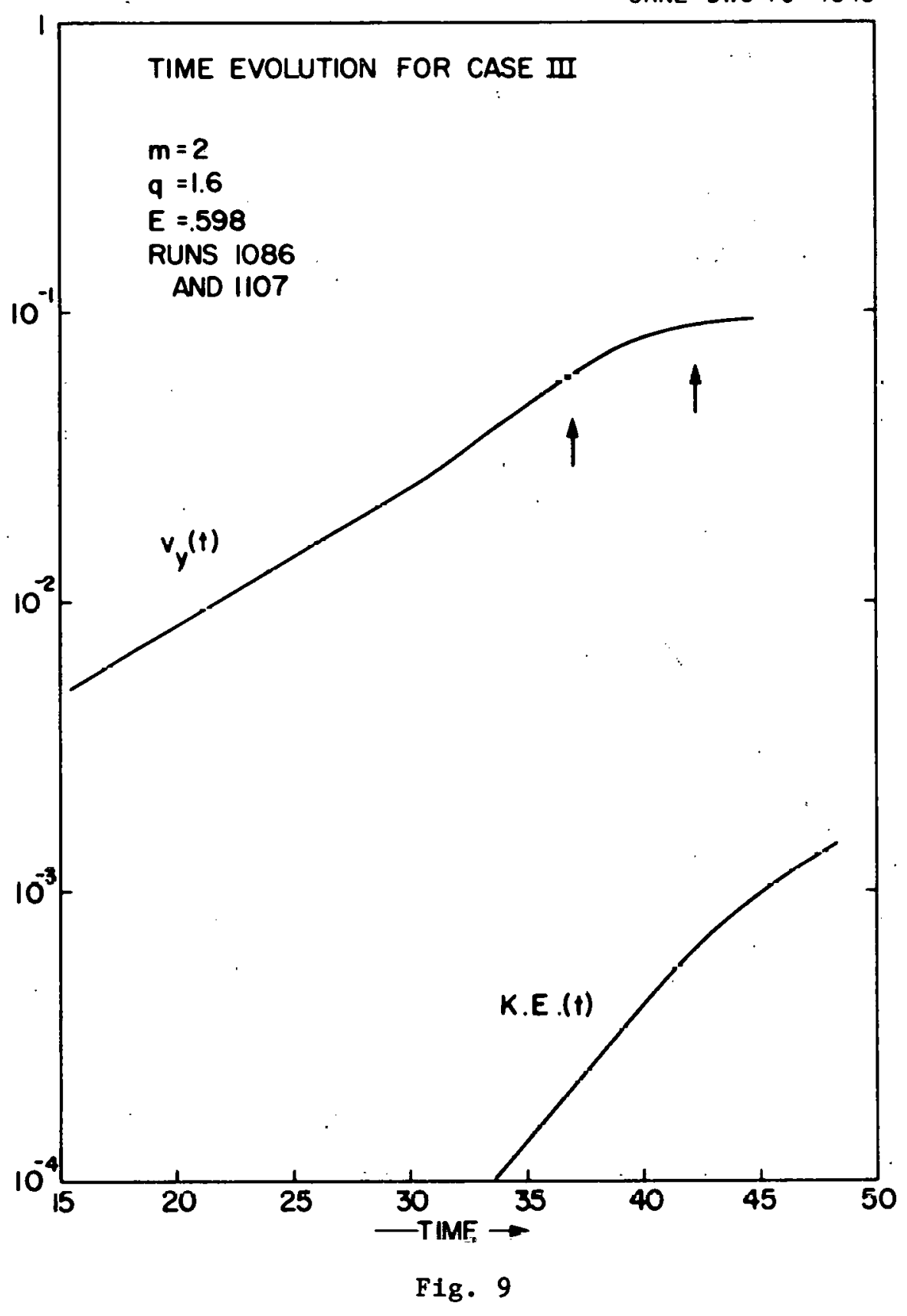




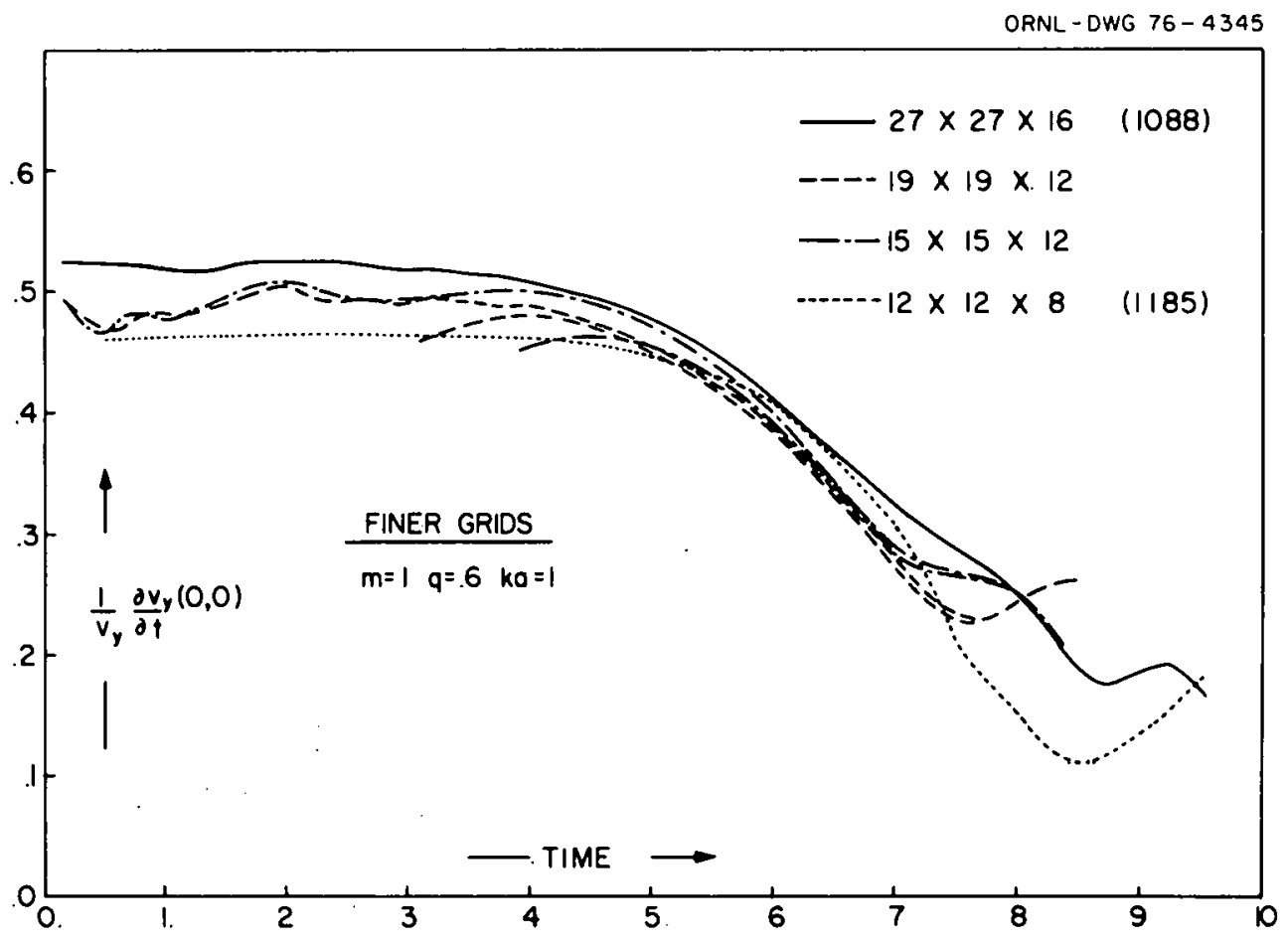

Fig. 10 


\section{THIS PAGE}

WAS INTENTIONALLY LEFT BLANK 


\section{APPENDIX I}

\section{Interpretation of Graphs}

Data are presented on cross sections of the plasma cylinder in the form of contour plots and arrow plots. Information needed to interpret these plots is presented here.

The contour plots are composed of five fields of numbers, each representing an interval of values logarithmically distributed over each decade. The interval $7.85 \leqslant x \leqslant 10.00$ is represented by 5 's, $4.83 \leqslant x$ $\leqslant 6.16$ by 4 's, $2.98 \leqslant x \leqslant 3.79$ by 3 's, $1.83 \leqslant x \leqslant 2.34$ by 2 's and $1.13 \leqslant x \leqslant 1.44$ by 1 's. The cycle repeats over the other decades - for example, the interval $78.50 \leqslant x \leqslant 100.00$ is again represented by 5 's. Space between the intervals is left blank. Negative numbers are represented by the letters A to E corresponding to the positive intervals labeled 1 to 5 , respectively.

The arrow plots are used to represent vector fields over a cross section. Arrows represent poloidal components while closed and open circles represent components into and out of the paper. The length of each arrow and the diameter of each circle represent the magnitudes of the respective components. The lengths are normalized so that the maximum value of the diameter or the $x$ - or $y$-component of any arrow is nine-tenths of the spacing between grid points. 
THIS PAGE

WAS INTENTIONALLY

LEFT BLANK 
ORNL/TM-5796

\section{.INTERNAL DISTRIBUTION}

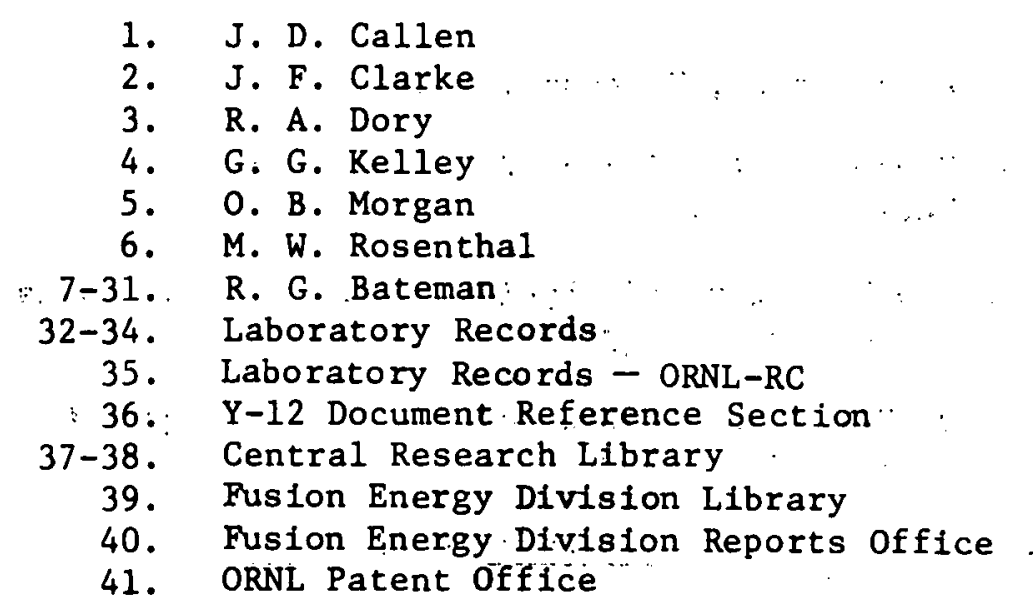

\section{EXTERNAL DISTRIBUTION}

42. Plasma Physics Library, Plasma Physics Laboratory, Princeton Univ., Forrestal Campus, P.O. Box 451, Princeton, NJ 08540

43. Controlled Thermonuclear Research Library, Lawrence Livermore Laboratory, P.0. Box 808, Livermore, CA 94550

44. Q Division Library, c/o F. L. Ribe, Los Alamos Scientific Laboratory, P.0. Box 1663, Los Alamos, NM 87544

45. Controlled Thermonuclear Research Library, c/o. Weston M. Stacey, Jr., Argonne National Laboratory, 9700 S. Cass Ave:, Argonne, IL 60439

46. CTR Computer Center, c/o Dr. John Killeen, Lawrence Livermore Laboratory, P.0. Box 808, Livermore, CA 94550

47. Librarian, Culham Laboratory, U.K. Atomic Energy Authority, Abingdon, Oxon, OX14 3DB, United Kingdom

48. Ruth Lengye, Bibliothek, Max-Planck Institut für Plasmaphysik, 8046 Garching bei München, Federal Republic of Germany

49. Library, Centre de Recherches en Physique des Plasmas, 21 Avenue des Bains, 1007, Lausanne, Switzerland

50. A. M. Dupas, : Documentation S.I.G.N., Départment de la Physiqué du Plasma et de la Fusion Controlée, Association EURATOM-CEA sur la Fusion, Centre d'Etudes Nucléaires, BP 85 Centre Du TRI 38041 Grenoble Cedex (France)

51. Bibliothèque, Service du Confinement des Plasmas, C.E.A., B.P. No. 6, 92, Fontenay-aux-Roses (Seine) France 
52. Library, International Centre for Theoretical Physics, Trieste, Italy

53. Library, Laboratorio Gas Ionizzati, Frascati, Italy

54. V. E. Ivanov, Physical-Technical Institute of the Ukranian Academy of Sciences, Sukhumi, U.S.S.R.

55. L. M. Kovrizhnikh, Lebedev Institute of Physics, Academy of Sciences of the U.S.S.R., Laninsky Prospect 53, Moscow, U.S.S.R.

56. Thermonuclear Laboratory, Kurchatov Institute of Atomic Energy, 46 Ulilsai Kulclialova, P.0. Box 3402, Moscow, U.3.3.R.

57. Library, Institute for Plasma Physics, Nagoya Univ., Nagoya, Japan $46^{\circ} 4$

58. Library, FOM-Institut voor Plasma-Fysica, Rijnhuizen, Jutphaas, Netherlands

59. Plasma Physics Group, Department of Engineering Physics, Australian National University, P.0. Box 4, Canberra A.C.T. 2600 Australia

60. Thermonuclear Library, Japan Atomic Energy Research Institute, Tokai, Naka, Ibaraki, Japan

61. Dr. D. G. McAlees, Exxon Nuclear Co., Inc., Research \& Technology Laser Enrichment Department, 2955 George Washington Way, Richland, WA .99352

62. CTR Reading Room, c/o Prof. D. W. Kerst, Dept. of Physics, Sterling Hall, Univ. of Wisconsin, Madison, WI 53706

63. CTR Reading Room, c/o Prof. I. B. Bernstein, Yale Univ., New llaven, CT 06510

64. Center for Plasma Physics and Thermonuclear Research, c/o D. W. Ross, Physics Dept., Univ. of Texas, Austin, TX 78712

65. CTR Reading Room, c/o Prof. B. D. Fried, Physics Dept., Univ, of California, Los Angeles, CA 90024

66. CTR Reading Room, c/o Prof. David C. Montgomery, Physics \& Astronomy Dept., Univ. of Iowa, Iowa City, IA 52240

67. Magneto-Fluid-Dynamics Library, Courant Inst. of Math. Sci., New York Univ., 251 Mercer St., New York, NY 10012

68. CTR Reading Room, c/o Prof. Allan N. Kaufman, Physics Dept., Univ. of California, Berkeley, CA 94720

69. CTR Reading Room, c/o Prof. W. B. Thompson, Physics Dept., Univ. of California, San Diego, La Jolla, CA 92037 
70. CTR Reading Room, c/o Prof: Alvin W. Trivelpiece; Dept. of Physics \& Astronomy, Univ. of Maryland, College Park, MD 20742

71: CTR Reading Room, c\% Prof: T. Kammash, 103 Research Admin. Bldg., N. Campus, Univ, of Michigan, Ann Arbor, MI 48105

72. CTR Reading Room, c\% Dr. Ravi N. Sudan, Phillips Hall, Cornell Univ., Ithaca, NY 14850

73. Prof. Marshall N. Rosenbluth, Institute for Advanced Study, Princeton, NJ 08540

74. CTR Reading Room, c/o Prof. R. Gross, Plasma Research Lab., Columbia Univ., New York, NY 10027

75. CTR Reading Room, c/o Prof. Roy Gould, California Inst. of Tech., M.S. 116-81, Pasadena, CA 91125

76." Dr. Nicholas A. Kràil, Science Applïcations, Inc., P.0. Box 2354, 1200 Prospect St., La Jollä, CA 92037

77. CTrR Reading Room, c/o Dr. Jay P. Boris, Plasma Physics, Naval Research Laboratory, Washington, DC 20390.

78. Professor A. Simon, Dept. of Mechanical \& Aerospace Sciences, University of Rochester, Rochester, NY 14627

79. CTR Library, "c/o Dr. Alan F. Haught, United Technologies Research Labs, East Hartford, CT 06108

80. Dr. Robert E. Price, Division of Magnetic Fusion Energy, G-234, Energy Research and Development Administration, Washington, DC 20545

81. Dr. R. C. Davidson, Division of Magnetic Fusion Energy, G-234, Energy Research and Development Administration, Washington, DC 20545

82. Dr. Arthur Sleeper, Division of Magnetic Fusion Energy, G-234, Energy Research" and Development Administration, Washington, DC 20545

83. Dr. Oscar P. Manley, Division of Magnetic Fusion Energy, G-234, Energy Research and Development Administration, Washington, DC 20545

$84:$ Dr. D. H. Priester, Division of Magnetic Fusion Energy, G-234, Energy Research and Development Administration, Washington, DC 20545 
86. Dr. L. D. Pearlstein, L-388, Lawrence Livermore Laboratory, P.0. Box 808, Livermore, CA 94550

87. Dr. J. P. Friedberg, Los Alamos Scientific Laboratory, Los Alamos NM 87544

88. Dr. David J. Rose, Dept. of Nuclear Engineering, MIT, Cambridge, MA 02139

89. Dr. Gareth E. Guest, General Atomic Co., P.0. Box 81608, San Diego, CA 92138

90. Dr. Claude Mercier, Service du Theorie des Plasmas, Centre d'Etudes Nucléaires, Fontenay-aux-Roses (Seine) France

91. Dr. J. B. Taylor, Culham Laboratory, UKAEA Abingdon, Oxon, OX14 3DB, United Kingdom

92. Dr. D. Pfirsch, Institute for Plasma Physics, 8046 Garching bei Mïinchen, Federal Republic of Germany

93. Dr. V. D. Shafranov, I. V. Kurchatov Inst. of Atomic Energy, 46 Ulitsa Kurchatova, P.O. Box 3402, Moscow, U.S.S.R.

94. Dr. Harold Grad, Courant Inst. of Math. Sci., New York Univ., 251 Mercer St., New York, NY 10012

95. Dr. J. G. Cordey, Cụlham Laboratory, UKAEA, Abingdon, Oxon, $0 \times 14$ 3DB, United Kingdom

96. Dr. David Baldwin, L-388, Lawrence Livermore Lab., P.0. Box 808, Livermore, CA. 94550

97. CTR Reading Room, c/o Prof. Bruno Coppi, Dept. of Physics, MIT, Cambridge, MA U213y

98. Dr. Harold P. Furth, Princeton Plasma Physics Lab., Princeton Unlv., P.0. Bux 451, Princeton, NJ 08540

99. Dr. Paul H. Kutherford, Princeton Plaswa Pliysics Lab., Princeton Univ., Princeton, NJ 08540

100. Dr. Herbert H. Woodson, Dept, of Electrical Engineering, Iniv. of Texas, Austin, TX 78712

101. Research \& Technical Support Div., Oak Ridge Operations, Energy Research and Development Administration, P. O. Box E, Oak Ridge, TN 37830

102-128. Tech. Information Center, Oak Ridge Operations, Energy Research and Development Administration, P. 0. Box 62, Oak Ridge, TN 37830 